\title{
Egalitarianism under earmark constraints
}

\author{
Olivier Bochet ${ }^{\mathrm{a}}$, Rahmi İlkılıç ${ }^{\mathrm{b}}$, Hervé Moulin ${ }^{\mathrm{c}, *}$ \\ ${ }^{a}$ University of Bern, Switzerland \\ ${ }^{\mathrm{b}}$ University of Bilkent, Turkey \\ ${ }^{\mathrm{c}}$ Department of Economics, Rice University, 6000 Main Street, Houston, TX 77005, USA
}

Received 2 September 2010; final version received 31 August 2012; accepted 14 September 2012

Available online 8 January 2013

\begin{abstract}
Agents with single-peaked preferences share a resource coming from different suppliers; each agent is connected to only a subset of suppliers. Examples include workload balancing, sharing earmarked funds, and rationing utilities after a storm.

Unlike in the one supplier model, in a Pareto optimal allocation agents who get more than their peak from underdemanded suppliers, coexist with agents who get less from overdemanded suppliers.

Our Egalitarian solution is the Lorenz dominant Pareto optimal allocation. It treats agents with equal demands as equally as the connectivity constraints allow. Together, Strategyproofness, Pareto Optimality, and Equal Treatment of Equals, characterize our solution.
\end{abstract}

(c) 2013 Elsevier Inc. All rights reserved.

JEL classification: C72; D63; D61; C78; D71

Keywords: Bipartite graph; Egalitarianism; Lorenz dominance; Single-peaked preferences; Strategyproofness

\footnotetext{
4 Acknowledgments: The authors warmly thank Anna Bogomolnaia, Jeremy Laurent-Lucchetti, and Jay Sethuraman for stimulating discussions, as well as two referees and the associate editor whose comments and insights greatly helped improve this paper. Olivier Bochet's research is supported by the Swiss National Fund (SNF) under grant 100014-126954. Part of the research was completed when Rahmi İlkılıç was affiliated with Maastricht University and he acknowledges the support of the European Community via Marie Curie Grant PIEF-GA-2008-220181. Hervé Moulin's research was supported by MOVE at the Universitat Autònoma de Barcelona.

* Corresponding author. Fax: +17133485278.

E-mail addresses: olivier.bochet@vwi.unibe.ch (O. Bochet), rahmi.ilkilic@bilkent.edu.tr (R. İlkıliç), moulin@rice.edu (H. Moulin).
} 


\section{Introduction}

Egalitarianism, the central principle of fair division, may conflict with incentives, feasibility or efficiency constraints. Maximizing the leximin ordering over profiles of relevant characteristics (a.k.a. the Rawlsian approach) is the most common implementation of egalitarianism under constraints. It is however a controversial method. Indeed, it recommends to take arbitrarily large amounts of resources from the "rich" if this allows to raise by even a tiny amount the lot of the "poor". The only case where egalitarianism eschews this critique is when we can find a Lorenz dominant distribution of welfare, or resources: at the Lorenz dominant outcome, we simultaneously maximize the share of the $k$ poorest individuals, for any number $k$ of agents. ${ }^{1}$ But unlike the leximin ordering that always reaches a unique maximum in any closed convex set, a Lorenz dominant outcome may not exist. We know very few fair division models admitting Lorenz dominating solutions over a reasonably rich domain of problems. The two main instances follow.

Dutta and Ray [11] observed that the core of a supermodular (convex) cooperative game is one general instance where a Lorenz dominant solution exists; this solution has been known after their work as the egalitarian selection in the core. The second model, due to Sprumont, is the fair division of a single commodity under single-peaked preferences and no free disposal [3,25]. The uniform solution selects for each agent either his peak or a common share, in such a way that the resource is fully distributed. Although the original motivation of the uniform solution was its incentive properties [3], its most compelling fairness property, and its shortest definition, is to be Lorenz dominant among all Pareto optimal allocations of the resource [10]. While the Lorenz dominance property of the Uniform solution is central in our paper, the solution satisfies many other compelling fairness criteria; see for instance [23] for an alternative characterization in terms of distributions of shares among agents.

Our contribution: We study a considerable generalization of the Sprumont model, where a homogeneous commodity (the resource) is still shared by several agents with single-peaked preferences, but the resource is coming from any number of different suppliers, under arbitrary bilateral feasibility constraints: each supplier can only deliver to a certain subset of agents. ${ }^{2}$ Consider a translation office which pays its employees a per page fee. Given the constant per page rate, each employee has a preferred amount of workload, which is private information to the agent. The total workload of the office is exogenous and must be allocated to the translators according to their publicly known language abilities: simplifying, each employee can handle a certain subset of languages. More generally the suppliers of our model can be different jobs, each one with a given size in work-hours, to be completed by a set of workers (the agents) with different skills, so each worker can only perform certain jobs. Another example involves the distribution of grants with earmark constraints: the resource is money, and there are several suppliers of funds (the granting agencies) to support different projects (the agents). Each agency must spend a certain budget, but the earmarks limit the set of projects that a given fund can support. $^{3}$ Or the resource can be water available from several sources, and geographical constraints limit the set of consumers (the agents) that a given source can serve. And so on.

The resources coming from different suppliers are, strictly speaking, different commodities, but any two commodities are perfect substitutes for an agent who can consume both. Thus we

\footnotetext{
1 References on Lorenz dominance: [24,16,13].

2 In the sequel, we sometimes refer to the Sprumont model as the standard model.

3 In United States politics, an earmark is a congressional provision that directs approved funds to be spent on specific projects.
} 
speak of a single commodity, like in the standard model, but of different resources (the suppliers in the above examples). Our agents have single-peaked preferences over the total amount of commodity they consume. We explore the implications of efficiency (Pareto Optimality), incentive-compatibility, and fairness in our bipartite model.

The set of Pareto optimal allocations has a much more complicated structure than in the standard model. There everyone consumes at most his peak if total demand exceeds the available resource, while everyone consumes at least his peak if total demand is smaller than the available resource. Here a Pareto optimal allocation involves typically some agents consuming more than their peak, and other agents consuming less. More precisely, the Pareto set is described by a three-components partition of the agents and resources: the first set of overdemanded resources are consumed exclusively by the first set of agents, who each receives at most their peak allocation; the second set of underdemanded resources are consumed exclusively by the second set of agents, who each receives at least their peak allocation; and the third set of balanced resources is allocated to the third set of agents, who each receives exactly their peak allocation.

We take Strategyproofness (truthful report of one's preferences is a dominant strategy) as our incentive compatibility design constraint. We identify the Egalitarian solution that generalizes the Uniform solution of the standard model. As in that model, this solution selects a Pareto optimal allocation, defines a Strategyproof direct revelation mechanism, and is fair in the strong sense that it selects the Lorenz dominant Pareto optimal allocation.

Our axiomatic characterization resembles closely that of the Uniform solution in [25,7]. Recall that in the standard model the Uniform solution is the unique solution that simultaneously satisfies Pareto Optimality, Strategyproofness and Equal Treatment of Equals - or No Envy. In our model the most restrictive interpretation of Equal Treatment of Equals says that two agents with identical preferences and access to the same sets of suppliers should receive the same allocation. We impose a stronger requirement, which does not hold agents responsible for their supply constraints. Our axiom Equal Treatment of Equal Demands says that two agents with identical preferences should get identical total shares, unless the feasibility constraints make this impossible (the precise definition is in Section 6). The alternative viewpoint is to hold agents responsible for theirs connections in the following sense: if Ann and Bob have identical preferences while Ann is able to consume more types of resources than Bob, she should end up with a bigger share. The manager of the translation office may want to treat trilingual Ann better than bilingual Bob, to reward Ann for having developed this additional skill; or she may view this as irrelevant to the distribution of the workload, unless of course the only available jobs are in the language that Ann masters but Bob does not. We take here the latter viewpoint, and refer the reader to [18] for a development of the former viewpoint in a bipartite model closely related to the current one. See also more comments in Section 6, when we formally define Equal Treatment of Equal Demands.

Our definition of No Envy takes a similarly neutral view on the pattern of compatibility constraints: Ann's envy of Bob's share matters even if she is less - or differently - connected than Bob. If it is feasible to improve Ann's share at the expense only of Bob, i.e., while preserving the shares of every agent other than Bob, then it is a legitimate objection ruled out by our No Envy axiom (Section 6).

The Egalitarian solution meets both properties above, and is characterized by the combination of Strategyproofness, Pareto Optimality and Equal Treatment of Equal Demands/No Envy, a result inspired by, and generalizing, the one in [7] for the standard model.

\section{Related literature:}

1) In a recent paper, Kar and Kibris [15] also consider the division of a single commodity coming from multiple suppliers. Every agent can consume from any supplier, but must receive 
his entire allocation from a single supplier: in effect, agents form coalitions to consume different private goods. It turns out that efficiency is incompatible with using a simple division rule, such as the Uniform rule, for every supplier.

2) Our characterization of the Pareto set is a consequence of the Gallai-Edmonds (henceforth, GE) decomposition for bipartite graphs (see [19]). This technique has already been applied to matching problems in [5] and [21].

3) The GE decomposition appears also in our companion paper [4], where we develop a model of bipartite trade in which both suppliers and demanders are active agents, in contrast to this paper where the supply side is inactive and must unload all the resources on active agents. In [4] each supplier (resp. demander) has single peaked preferences over the amount of commodity he wants to supply (resp. receive); the homogeneous commodity can only be transferred across certain bilateral edges. The set of relevant Pareto optimal allocations is described by the same GE decomposition as here, it contains again a Lorenz dominant allocation, which defines a strategyproof direct revelation mechanism. However the two models differ in important ways. The mechanism in [4] ensures Voluntary Participation by all agents, therefore no supplier (or demander) ever gets to supply (or receive) more than her ideal level. In the current model, the underdemanded suppliers unload all their endowment to agents who end up with more than their peak consumption. By contrast in [4] these suppliers are rationed (cannot unload their peak supply) while the corresponding demanders consume their peak allocation. On the other hand the overdemanded suppliers here become the suppliers who supply their peak load in [4], and the corresponding demanders are rationed in [4] exactly as they are here. Thus an allocation problem in [4] amounts to the concatenation of two problems here, each with one overdemanded side and the other side rationed (consuming below peak).

Another difference arises when we consider the impact of destroying one edge in the bipartite graph of compatibility constraints. In [4] this is (weakly) detrimental to the agents at both ends of the edge. By contrast, in our model, dropping an edge can be good or bad news for the agent at one end of this edge, as well as to other agents: this is explained in the concluding Section 8. It implies that if the edges are not verifiable (agents can freely claim to be incompatible with some suppliers) our solution is vulnerable to manipulation, while the mechanism in [4] is not.

We note finally that the proofs in [4] rely on the techniques of flows on graphs, in particular the max-flow min-cut theorem, instead of the GE decomposition we use here. The flow approach simplifies some proofs there, and could have been used here as well, at some notational cost.

4) We conclude with three follow up papers. Chandramouli and Sethuraman [6] establish that our Egalitarian solution is actually Group-Strategyproof, thus answering an open question in an earlier version of this paper (see Proposition 4 in Section 6).

Szwagrzak [26] obtains an alternative characterization of the Egalitarian solution where instead of Strategyproofness he uses a bipartite version of the Preference Replacement property, that is the basis of an alternative characterization of the Uniform rule in the standard model (Thomson [28]).

Finally, Moulin and Sethuraman [18] discuss the extension to our bipartite model of other solutions of the standard rationing problem, such as the Proportional and Equal Losses solutions. They assume complete information of demands and supplies, and take the viewpoint that agents are responsible for their own connections. They generalize the familiar Consistency property to the bipartite context. Consistency in our model is briefly discussed in comment 4 of Section 8 .

Contents: After a numerical example in the next section, we introduce the model in Section 3 , and characterize Pareto optimal allocations in Section 4. The Egalitarian solution is defined in Section 5 and its fairness and incentives properties are the subject of Section 6. Section 7 contains 


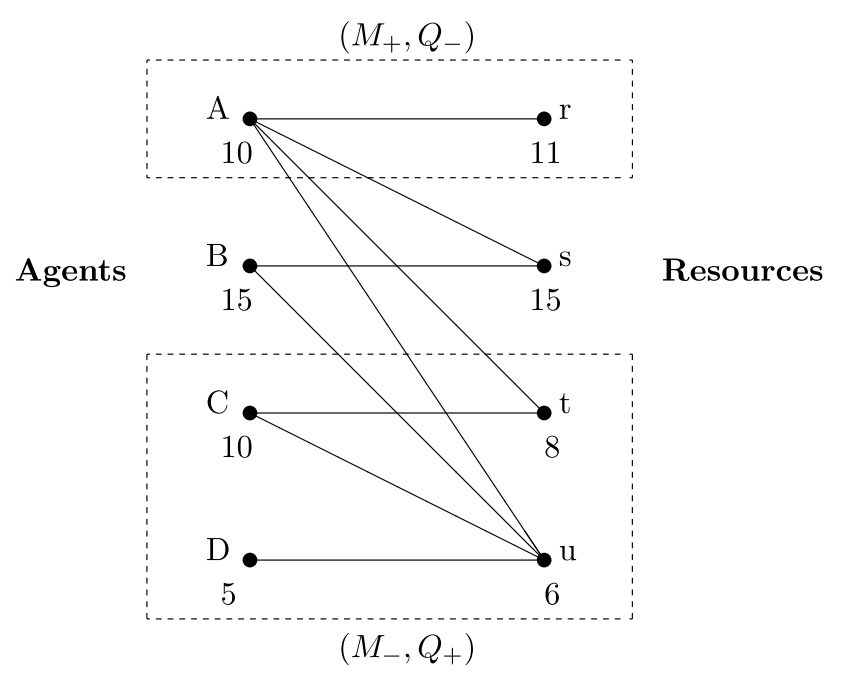

Fig. 1. A bipartite problem and its canonical decomposition.

the characterization result, and Section 8 collects final comments about variants and possible extensions of our model.

\section{A numerical example}

We have four resources $r, s, t, u$, and four agents $A, B, C, D$. Fig. 1 shows the compatibility constraints: e.g., agent $A$ can consume from any resource, while agent $D$ can only consume from resource $u$. Total supply $11+15+8+6=40$ equals total demand $10+15+10+5=40$. In the standard model, this would allow to give every agent her peak allocation. The bipartite constraints do not allow this: $A$, for instance, must consume at least 11 units, because no one else can consume the first resource. On the other hand, $D$ should not consume more than 5 units in a Pareto optimal allocation: for instance if we give him 6 units, $C$ can get at most 8 units, and transferring one unit of resource $u$ from $D$ to $C$ is a Pareto improvement.

The three-component partition identifies $r$ as the underdemanded resource, and $A$ as the agent consuming exclusively the $r$-resource. There are two overdemanded resources $t$ and $u$, whose resources go exclusively to $C$ and $D$. Finally resource $s$ is balanced with agent $B$. By Proposition 1 below, an allocation is Pareto optimal if and only if: $A$ gets 11 units from $r ; B$ gets 15 from $s$; $D$ gets $\theta$ units from $u$, where $4 \leqslant \theta \leqslant 5$, and $C$ gets all of $t$ plus $(6-\theta)$ from $u$. This guarantees that each one of $C, D$ gets no more than his peak.

Within the Pareto set, our Egalitarian solution picks the most equal feasible set of shares, corresponding to $\theta=5$ : $C$ gets 9 units and $D$ gets 5 .

\section{The model}

We have a set $M$ of agents with generic elements $i, j, k, \ldots$, and $m=|M|$; and a set $Q$ of resources with generic element $r, s, \ldots$, and $q=|Q|$. Resource $r$ is of size $\omega_{r}$, where $\omega_{r}>0$.

All resources must be allocated between the agents, but each resource can only be assigned to some of the agents. The bipartite graph $G$, a subset of $M \times Q$, represents the compatibility 
constraints between resources and agents: ir $\in G$ means that it is possible to transfer resource $r$ to agent $i$. We assume throughout that the graph $G$ is connected, else we can treat each connected component of $G$ as a separate problem.

We use the following notation: for any subsets $S \subseteq M, T \subseteq Q$ the restriction of $G$ is $G(S, T)=G \cap\{S \times T\}$ (not necessarily connected); the set of resources compatible with agents in $S$ is $f(S)=\{r \in Q \mid G(S,\{r\}) \neq \emptyset\}$, the set of agents compatible with resources in $T$ is $g(T)=\{i \in M \mid G(\{i\}, T) \neq \emptyset\}$.

A transfer of resources from $Q$ to $M$ is described by a $G$-flow $\varphi$, i.e., a vector $\varphi \in \mathbb{R}_{+}^{G}$ such that $\varphi_{i r}>0 \Rightarrow \operatorname{ir} \in G$. We call a $G$-flow $\varphi$ feasible if it allocates all the resources and we write $x(\varphi)$ for the allocation it realizes:

$$
\text { for all } r \in Q: \quad \sum_{i \in g(r)} \varphi_{i r}=\omega_{r} ; \quad \text { for all } i \in M: \quad x_{i}(\varphi)=\sum_{r \in f(i)} \varphi_{i r}
$$

We write $\mathcal{F}(G ; \omega)$ for the set of feasible $G$-flows, and $\mathcal{A}(G, \omega)=x(\mathcal{F}(G ; \omega))$ for the set of allocations achieved by some feasible $G$-flow. Both sets are obviously nonempty.

Agent $i$ has single-peaked preferences over her total share of resources. A single-peaked preference $R_{i}$ is transitive and complete over the nonnegative real line $\mathbb{R}_{+}$. The symmetric and asymmetric parts of $R_{i}$ are denoted by $I_{i}$ and $P_{i}$, respectively. Preference $R_{i}$ has a "peak" $p\left[R_{i}\right] \in \mathbb{R}_{+}$such that for each $x_{i}, x_{i}^{\prime} \in \mathbb{R}_{+}$

$$
\begin{aligned}
& x_{i}^{\prime}<x_{i} \leqslant p\left[R_{i}\right] \Rightarrow x_{i} P_{i} x_{i}^{\prime} \\
& p\left[R_{i}\right] \leqslant x_{i}<x_{i}^{\prime} \Rightarrow x_{i} P_{i} x_{i}^{\prime}
\end{aligned}
$$

Let $\mathcal{R}$ be the set of single-peaked preferences over $\mathbb{R}_{+}$. A preference profile is then $R=$ $\left(R_{i}\right)_{i \in M} \in \mathcal{R}^{M}$. For each $R \in \mathcal{R}^{M}$, we let $p[R]=\left(p\left[R_{i}\right]\right)_{i \in M}$ be the associated profile of peaks. Several of our definitions and results bear on a fixed profile $R$; thus, whenever this causes no confusion, we simply write $p_{i}$ in place of $p\left[R_{i}\right]$ and $p$ in place of $p[R]$.

An economy is a triple $(G, \omega, R)$.

We use the following notation throughout: for any finite sets $D, E$ such that $D \subset E$, and any vector $t \in \mathbb{R}^{E}$, we write $t_{D}=\sum_{e \in D} t_{e}$, and $t^{D}$ for the projection of $t$ on $\mathbb{R}^{D}$.

\section{Feasible and Pareto optimal allocations}

We describe first the set $\mathcal{A}(G, \omega)$ of feasible allocations.

The allocation $x \in \mathbb{R}^{M}$ is in the lower (resp. upper) core of the cooperative game $(M, w)$ if $x_{M}=w(M)$, and $x_{S} \geqslant w(S)$ (resp. $\left.x_{S} \leqslant w(S)\right)$ for all $S \subseteq M$. The game $(M, w)$ is supermodular (resp. submodular) if $w(S)+w(T) \leqslant w(S \cup T)+w(S \cap T)$ (resp. $\geqslant$ ) for all $S, T \subseteq M$.

Lemma 1 (Feasible allocations). The following statements are equivalent:

i) $x \in \mathcal{A}(G, \omega)$;

ii) for all $S \subseteq M, x_{S} \leqslant \omega_{f(S)}$ and $x_{M}=\omega_{Q}$;

iii) for all $T \subseteq Q, \omega_{T} \leqslant x_{g(T)}$ and $\omega_{Q}=x_{M}$;

iv) $x$ is in the lower core of the supermodular TU game $(M, w)$, where

$$
w(S)=\max _{T: g(T) \subseteq S}\left\{\omega_{T}\right\}
$$


v) $x$ is in the upper core of the submodular TU game $(M, v)$, where

$$
v(S)=\omega_{f}(S)
$$

Proof. By a standard continuous version of the Marriage Lemma (a.k.a. Hall's theorem [14], see [1]), i) is equivalent to either of ii) or iii).

Statements ii) and v) are clearly equivalent.

We show finally that statements iv), v) are equivalent. Because $G$ is connected we have $w(M)=\omega_{Q}$, and $v(M)=\omega_{Q}$. We let the reader check that $(M, w)$ and $(M, v)$ are dual games, i.e., $w(S)+v(M \backslash S)=\omega_{Q}$ for all $S$. The inequality $\omega_{f(S)}+\omega_{f\left(S^{\prime}\right)} \geqslant \omega_{f\left(S \cup S^{\prime}\right)}+\omega_{f\left(S \cap S^{\prime}\right)}$ follows from $f(S) \cup f\left(S^{\prime}\right)=f\left(S \cup S^{\prime}\right)$ and $f(S) \cap f\left(S^{\prime}\right) \supseteq f\left(S \cap S^{\prime}\right)$. It implies that $(M, v)$ is submodular. Hence the dual game $(M, w)$ is supermodular. The equivalence follows by duality again.

An allocation $x \in \mathcal{A}(G, \omega)$ is Pareto optimal in the economy $(G, \omega, R)$ if for any other $x^{\prime}$ in $\mathcal{A}(G, \omega)$, we have

$$
\left\{x_{i}^{\prime} R_{i} x_{i} \text { for all } i \in M\right\} \Rightarrow\left\{x_{i}^{\prime} I_{i} x_{i} \text { for all } i \in M\right\}
$$

We write $\mathcal{P O}(G, \omega, R)$ for the set of Pareto optimal allocations. The structure of this set is given by a three-component partition of $M \cup Q$ that we derive as a variant of the classic GallaiEdmonds decomposition for bipartite graphs [19]. This partition, as well as the $\operatorname{set} \mathcal{P} \mathcal{O}(G, \omega, R)$ itself, depend only upon the profile of peaks $p[R]$, but not on the way $R_{i}$ compares allocations across $p_{i}$.

We call a triple $(G, \omega, p)$ a problem, keeping in mind it represents all economies where $p$ is the profile of peaks in $R$. Until the end of Section 5 it is enough to work with problems rather than economies. We define three properties of a problem:

- $(G, \omega, p)$ is balanced if $p \in \mathcal{A}(G, \omega)$;

- $(G, \omega, p)$ exhibits underdemand if for all $S \subseteq M, p_{S}<\omega_{f(S)}$;

- $(G, \omega, p)$ exhibits overdemand if for all $T \subseteq Q, \omega_{T}<p_{g(T)}$.

In a balanced problem we can give exactly his peak allocation to every agent. By Lemma 1 in a problem with underdemand we can give each agent at least his peak, and must give strictly more to at least one agent; in a problem with overdemand we can give each agent at most his peak, and must give strictly less to at least one.

We show now that any allocation problem $(G, \omega, p)$ can be decomposed in three subproblems, one of each type above, and at most two types may be absent. When we speak of the subproblem restricted to $S \times T \subseteq M \times Q$, we mean that the resources in $T$ must be assigned to the agents in $S$ along the restricted graph $G(S, T)$ (which may not be connected), i.e. we talk about the subproblem $\left(G(S, T), \omega^{T}, p^{S}\right)$ and the set of feasible allocations $\mathcal{A}\left(G(S, T), \omega^{T}\right)$.

Lemma 2. For any problem $(G, \omega, p)$ where $G$ is connected, and $p \geqslant 0, \omega \gg 0$, there exist unique partitions $M_{+}, M_{0}, M_{-}$of $M$, and $Q_{+}, Q_{0}, Q_{-}$of $Q$ such that

i) $G\left(M_{-}, Q_{0}\right)=G\left(M_{-}, Q_{-}\right)=G\left(M_{0}, Q_{-}\right)=\emptyset$;

ii) $\left(G\left(M_{+}, Q_{-}\right), \omega^{Q_{-}}, p^{M_{+}}\right)$exhibits underdemand;

iii) $\left(G\left(M_{0}, Q_{0}\right), \omega^{Q_{0}}, p^{M_{0}}\right)$ is balanced;

iv) $\left(G\left(M_{-}, Q_{+}\right), \omega^{Q_{+}}, p^{M_{-}}\right)$exhibits overdemand. 
We repeat that up to two of the pairs $\left(M_{+}, Q_{-}\right),\left(M_{0}, Q_{0}\right)$, or $\left(M_{-}, Q_{+}\right)$may be empty. For instance, if there are no bilateral constraints $(G=M \times Q)$, our model is a generalization of Sprumont's [25], where the GE decomposition reduces to a single component: if $\omega_{Q}<p_{M}$ we have overdemand, $M=M_{-}, Q=Q_{+}$; if $\omega_{Q}>p_{M}$ underdemand and $M=M_{+}, Q=Q_{-}$; if $\omega_{Q}=p_{M}$ the problem is balanced and $M=M_{0}, Q=Q_{0}$. See the discussion of this special case after Proposition 2 in Section 5.

Proof of Lemma 2. Constructing the partitions. Fix $\left(G(M, Q), \omega^{Q}, p^{M}\right)$ and define the partitions with the help of two simple maximization problems. Set $\mathcal{D}=\arg \max _{S \subseteq M}\left\{p_{S}-\omega_{f(S)}\right\}$ if there is at least one $S$ such that $p_{S}>\omega_{f(S)}, \mathcal{D}=\emptyset$ otherwise. As $S \rightarrow p_{S}-\omega_{f(S)}$ is supermodular, the set $\mathcal{D}$ is closed under intersection and union. If $\mathcal{D} \neq \emptyset$, we define $M_{-}$as its smallest element, and $M_{-} \cup M_{0}$ as its largest element. Set similarly $\mathcal{B}=\arg \max _{T \subseteq Q}\left\{\omega_{T}-p_{g(T)}\right\}$ if there is at least one $T$ such that $\omega_{T}>p_{g(T)}, \mathcal{B}=\emptyset$ else. Then if $\mathcal{B} \neq \emptyset$, it is closed under intersection and union, and $Q_{-}$is its smallest element, while $Q_{-} \cup Q_{0}$ is its largest element.

Proof of property $i)$. Suppose first that $\mathcal{D}$ and $\mathcal{B}$ are both nonempty, and check $G\left(M_{-}, Q_{-} \cup\right.$ $\left.Q_{0}\right)=\emptyset$ by contradiction. If this set is nonempty, we define $A=g\left(Q_{-} \cup Q_{0}\right) \cap M_{-}$, and $B=$ $f\left(M_{-}\right) \cap\left\{Q_{-} \cup Q_{0}\right\}$, so that $A=g(B) \cap M_{-}$and $B=f(A) \cap\left\{Q_{-} \cup Q_{0}\right\}$. Consider the equality

$$
\left(p_{M_{-} \backslash A}-\omega_{f\left(M_{-}\right) \backslash B}\right)+\left(p_{A}-\omega_{B}\right)=p_{M_{-}}-\omega_{f\left(M_{-}\right)}
$$

By construction $f\left(M_{-} \backslash A\right) \subseteq f\left(M_{-}\right) \backslash B$, therefore the inequality $p_{M_{-} \backslash A}-\omega_{f\left(M_{-}\right) \backslash B} \geqslant$ $p_{M_{-}}-\omega_{f\left(M_{-}\right)}$would imply that $M_{-} \backslash A$ is a smaller element of $\mathcal{D}$ than $M_{-}$. Thus we must have $p_{A}-\omega_{B}>0$. Consider now the equality

$$
\left(\omega_{\left\{Q_{-} \cup Q_{0}\right\} \backslash B}-p_{g\left(Q_{-} \cup Q_{0}\right) \backslash A}\right)+\left(\omega_{B}-p_{A}\right)=\omega_{Q_{-} \cup Q_{0}}-p_{g}\left(Q_{-} \cup Q_{0}\right)
$$

By construction $g\left(\left\{Q_{-} \cup Q_{0}\right\} \backslash B\right) \subseteq g\left(Q_{-} \cup Q_{0}\right) \backslash A$; combining this with $\omega_{B}-p_{A}<0$, we see that $T=\left\{Q_{-} \cup Q_{0}\right\} \backslash B$ gives a higher $\omega_{T}-g(T)$ than $Q_{-} \cup Q_{0}$, the desired contradiction.

A symmetrical argument, omitted for brevity, establishes $G\left(M_{-} \cup M_{0}, Q_{-}\right)=\emptyset$.

Next we define $Q_{+}=f\left(M_{-}\right)$, and check that $Q_{+}, Q_{0}, Q_{-}$partition $Q$. We already know that these sets are disjoint. If they are not a partition, the set $T=Q \backslash\left\{Q_{+} \cup Q_{-} \cup Q_{0}\right\}$ is nonempty. Because $T \cup\left\{Q_{-} \cup Q_{0}\right\}$ is not in $\mathcal{B}$, we have $p_{g(T) \backslash g\left(Q_{-} \cup Q_{0}\right)}>\omega_{T}$. Therefore the set $S=$ $g(T) \backslash g\left(Q_{-} \cup Q_{0}\right)$ is nonempty. Moreover $S$ and $M_{-}$are disjoint and $f\left(M_{-} \cup S\right) \subseteq Q_{+} \cup T$. This gives a contradiction of the definition of $M_{-}$:

$$
p_{M_{-} \cup S}-\omega_{f\left(M_{-} \cup S\right)} \geqslant p_{M_{-} \cup S}-\omega_{Q_{+} \cup T}=p_{M_{-}}-\omega_{Q_{+}}+p_{S}-\omega_{T}>p_{M_{-}}-\omega_{Q_{+}}
$$

We omit the symmetrical argument establishing that $M_{+}=g\left(Q_{-}\right)$completes the partition of $M$ as $M_{+}, M_{0}, M_{-}$.

Proof of property iv). To check the overdemand in $\left(G\left(M_{-}, Q_{+}\right), \omega^{Q_{+}}, p^{M_{-}}\right)$recall that $\omega_{Q_{+}}<p_{M_{-}}$because $\mathcal{D}$ is nonempty. Then fix a proper subset $T$ of $Q_{+}$and assume $\omega_{T} \geqslant$ $p_{g(T) \cap M_{-}}$. The equality

$$
\left(p_{M_{-} \backslash g(T)}-\omega_{Q_{+} \backslash T}\right)+\left(p_{g(T) \cap M_{-}}-\omega_{T}\right)=p_{M_{-}}-\omega_{Q_{+}}
$$

implies that $M_{-} \backslash g(T)$ is a smaller element of $\mathcal{D}$ than $M_{-}$, contradiction.

Proof of properties iii) and ii). The proof that $\left(G\left(M_{0}, Q_{0}\right), \omega^{Q_{0}}, p^{M_{0}}\right)$ is balanced proceeds along similar lines. If there is $S \subseteq M_{0}$ such that $p_{S}>\omega_{f(S) \cap Q_{0}}$, it follows that $S^{\prime}=M_{-} \cup S$ gives a higher $p_{S^{\prime}}-\omega_{f\left(S^{\prime}\right)}$ than $M_{-}$. We omit the details.

We also omit for brevity the symmetrical proof that $\left(G\left(M_{+}, Q_{-}\right), \omega^{Q_{-}}, p^{M_{+}}\right)$is underdemanded, and the treatment of the remaining cases where at least one of $\mathcal{D}$ or $\mathcal{B}$ is empty. For 


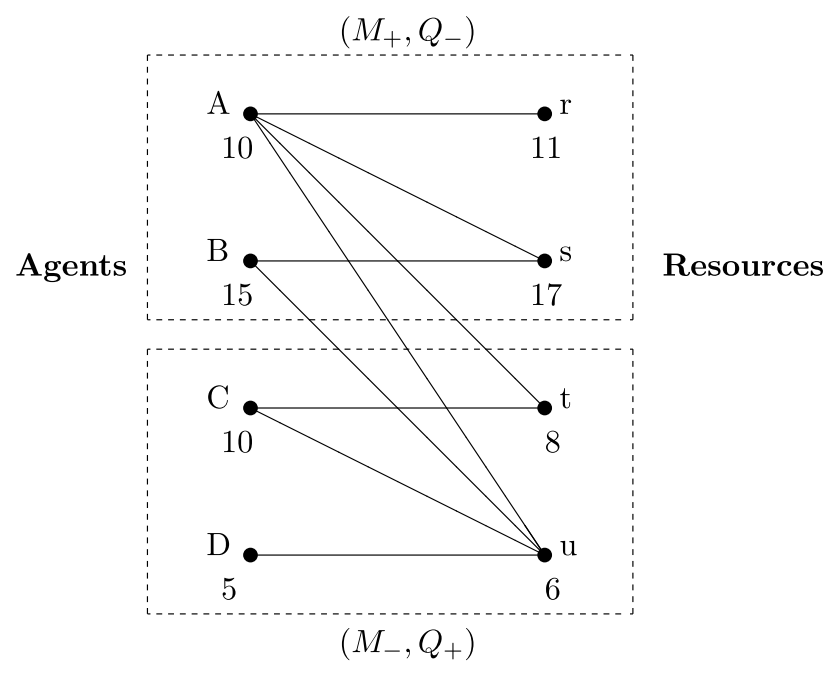

Fig. 2. Sensitivity of the Gallai-Edmonds decomposition.

instance if they are both empty we have $p_{S} \leqslant \omega_{f(S)}$ and $\omega_{T} \leqslant p_{g(T)}$ for all $S \subseteq M, T \subseteq Q$, and Lemma 1 implies that $(G, \omega, p)$ is balanced; so $M=M_{0}$ and $Q=Q_{0}$.

The above proof shows that the canonical partition obtains by maximizing two supermodular set functions, one over the subsets of $M$, the other those of $Q$. This can be done by the standard greedy algorithm, ${ }^{4}$ of polynomial complexity in the number of nodes $|M|+|Q|$ (see for instance Chapter 16 in [8]).

We already described the partition in the example of Section 2: $M_{+}=\{A\}, M_{0}=\{B\} ; M_{-}=$ $\{C, D\} ; Q_{-}=\{r\} ; Q_{0}=\{s\} ; Q_{+}=\{t, u\}$. For another example consider a variant of Fig. 1 in which the only change is that the resource $s$ is of size 17 instead of 15. See Fig. 2. Now the canonical partition has only two components: $r, s$ are the underdemanded resources, while $t, u$ remain overdemanded, i.e., $M_{+}=\{A, B\} ; M_{-}=\{C, D\} ; Q_{-}=\{r, s\} ; Q_{+}=\{t, u\}$.

Figs. 1 and 2 illustrate a general property, an immediate consequence of Lemma 2: for any pair of agents $i, j$ such that $f(i) \subseteq f(j)$, we have $\left\{j \in M_{-} \Rightarrow i \in M_{-}\right\}$and $\left\{i \in M_{+} \Rightarrow j \in M_{+}\right\}$, and if two resources $r, s$ satisfy $g(r) \subseteq g(s)$, then $\left\{s \in Q_{-} \Rightarrow r \in Q_{-}\right\}$and $\left\{r \in M_{+} \Rightarrow s \in M_{+}\right\}$.

Our first proposition uses the familiar meta-property Peak-Only that we now define. Given some property $X$, let $\mathcal{X}(G, \omega, R)$ be the set of allocations that satisfy property $X$ in economy $(G, \omega, R)$.

Peak-Only. Fix an economy $(G, \omega, R)$. Property $X$ is Peak-Only if for each $R^{\prime} \in \mathcal{R}^{M}$ with $p[R]=p\left[R^{\prime}\right]$, we have $\mathcal{X}(G, \omega, R)=\mathcal{X}\left(G, \omega, R^{\prime}\right)$.

We describe now the set $\mathcal{P O}(G, \omega, R)$ of Pareto optimal allocations in terms of the canonical decomposition of Lemma 2.

\footnotetext{
4 If $S \rightarrow v(S)$ is supermodular over $2^{M}$, we solve first $\max _{i \in M} v(i)$, keep a winner $i^{*}$, then solve $\max _{i \in M \backslash\left\{i^{*}\right\}} v\left(\left\{i, i^{*}\right\}\right)$, and so on.
} 
From now on we write a subproblem such as $\left(G\left(M_{+}, Q_{-}\right), \omega^{Q_{-}}, p^{M_{+}}\right)$simply as $\left(G\left(M_{+}, Q_{-}\right), \omega, p\right)$, and the feasible allocation set $\mathcal{A}\left(G(S, T), \omega^{T}\right)$ as $\mathcal{A}(G(S, T), \omega)$. This will cause no confusion if we keep in mind that only the coordinates of $\omega$ in $Q_{-}, T$, etc., matter.

\section{Proposition 1. Fix $G$ and $\omega$.}

i) For any profile $R \in \mathcal{R}^{M}$ with peaks $p$, the set $\mathcal{P O}(G, \omega, R)$ is nonempty, convex and compact;

ii) The property of Pareto Optimality is Peak-Only;

iii) A flow implementing a Pareto optimal allocation is null on $G\left(M_{+}, Q_{+}\right), G\left(M_{+}, Q_{0}\right)$, and $G\left(M_{0}, Q_{+}\right)$

iv) The allocation $x$ is Pareto optimal if and only if

$$
\begin{aligned}
& \text { in } M_{+}: \quad x^{M_{+}} \in \mathcal{A}\left(G\left(M_{+}, Q_{-}\right), \omega\right), \quad \text { and } \quad p^{M_{+}} \leqslant x^{M_{+}} \\
& \text {in } M_{0}: \quad x^{M_{0}}=p^{M_{0}} \\
& \text { in } M_{-}: \quad x^{M_{-}} \in \mathcal{A}\left(G\left(M_{-}, Q_{+}\right), \omega\right), \quad \text { and } \quad x^{M_{-}} \leqslant p^{M_{-}}
\end{aligned}
$$

In words, agents in $M_{+}$consume precisely all the resources in $Q_{-}$, each one gets at least his peak, and at least one, strictly more (Lemma 2); those in $M_{-}$share the resources in $Q_{+}$, consume no more than their peak, and at least one gets strictly less; those in $M_{0}$ consume the resources in $Q_{0}$ and each one gets precisely his peak.

The Peak-Only property allows us to write the Pareto optimal set simply as $\mathcal{P O}(G, \omega, p)$. From statements iv) and v) in Lemma 1, we can also describe $\mathcal{P O}(G, \omega, p)$ as the cartesian product of three sets: in $M_{+}$, the subset of the upper core of $\left(M_{+}, v^{+}\right)$such that $x^{M_{+}} \geqslant p^{M_{+}}$, where $v^{+}$is the game (3) for the restricted problem $\left(G\left(M_{+}, Q_{-}\right), \omega, p\right)$; in $M_{-}$the subset of the lower core of $\left(M_{-}, w^{-}\right)$such that $x^{M_{-}} \leqslant p^{M_{-}}$, where $w^{-}$is the game (2) for $\left(G\left(M_{-}, Q_{+}\right), \omega, p\right)$; and $p^{M_{0}}$ in $M_{0}$.

Proof of Proposition 1. Note first that statements i) and ii) follow at once from statement iv).

Step 1. Proving the "if" part of statement iv). By statement ii) in Lemma 1, and the fact that $\left(G\left(M_{+}, Q_{-}\right), \omega, p\right)$ exhibits underdemand (Lemma 2), the set $\mathcal{A}\left(G\left(M_{+}, Q_{-}\right), \omega\right)$ is nonempty; the set $\mathcal{A}\left(G\left(M_{-}, Q_{+}\right), \omega\right)$ is similarly nonempty because $\left(G\left(M_{-}, Q_{+}\right), \omega, p\right)$ exhibits overdemand. Finally, $\mathcal{A}\left(G\left(M_{0}, Q_{0}\right), \omega, p\right)$ is nonempty because $\left(G\left(M_{0}, Q_{0}\right), \omega, p\right)$ is balanced. Suppose now that an allocation $x$ satisfying (4), (5), (6) is Pareto dominated by some $y \in \mathcal{A}(G, \omega)$. Clearly we have $y^{M_{0}}=x^{M_{0}}$. Because $G\left(M_{-} \cup M_{0}, Q_{-}\right)=\emptyset$ we have $y_{M_{+}} \geqslant \omega_{Q_{-}}=x_{M_{+}}$; on the other hand if $y_{i}>x_{i}$ for some $i \in M_{+}$, this agent with peak $p_{i} \leqslant x_{i}$ strictly prefers $x_{i}$ to $y_{i}$ which our assumption precludes. We conclude $y^{M_{+}}=x^{M_{+}}$. The argument establishing $y^{M_{-}}=x^{M_{-}}$is entirely similar.

Step 2. Proving the "only if" part of statement iv), and statement iii). We fix throughout Step 2 an economy $(G, \omega, R)$, a Pareto optimal allocation $x$, and a flow $\varphi$ implementing $x$. We color agent $i$ in green if $x_{i}<p_{i}$, in red if $x_{i}>p_{i}$, and in black if $x_{i}=p_{i}$. We also construct a directed graph $G^{\varphi}$ as follows: all edges in $G$ are oriented from $M$ to $Q$; if $\varphi_{i j}>0$, and only then, we add a "backward" edge from resource $j$ to agent $i$. 
We claim there is no directed path in $G^{\varphi}$ from a green agent to a red one. ${ }^{5}$ If there was such a path from $i$ to $i^{\prime}$, we could increase a little the flow along that path (with the convention that increasing the flow on a backward edge amounts to decrease by the same amount the flow $\varphi_{i j}$ in $G$ ), and obtain a new allocation where $i$ consumes a little more, $i^{\prime}$ a little less, and everyone else as before; this would contradict Pareto Optimality.

Define now $X$ as the set of all green nodes in $M$ together with the nodes in $M \cup Q$ that one can reach in $G^{\varphi}$ from a green node; $Y$ as the set of nodes in $M \cup Q$ that are either a red agent, or a node from which one can reach a red node in $G^{\varphi}$; and $Z$ as the remaining subset of $M \cup Q$. Thus $X, Y, Z$ partition $M \cup Q$, and every agent in $X \cap M$ (resp. $Y \cap M$, resp. $Z \cap M$ ) is green or black (resp. red or black, resp. black). Also, there is no path in $G^{\varphi}$ from $X$ to $Z$ or $Y$, or from $Z$ to $Y$.

Substep 2.1. In this substep we focus on $M_{-}$and $Q_{+}$and show $M_{-}, Q_{+} \subseteq X$, in particular there is no red agent in $M_{-}$, and $x^{M_{-}} \leqslant p^{M_{-}}$.

Assume to the contrary $(Y \cup Z) \cap M_{-} \neq \emptyset$. Then $x_{(Y \cup Z) \cap M_{-}} \geqslant p_{(Y \cup Z) \cap M_{-}}$, because all such agents are red or black. We also have $x_{(Y \cup Z) \cap M_{-}} \leqslant \omega_{(Y \cup Z) \cap Q_{+}}$, because the only positive flow out of $(Y \cup Z) \cap M_{-}$goes to $(Y \cup Z) \cap Q_{+}$: it cannot go to $X$ without creating a path in $G^{\varphi}$ from $X$ to $Y \cup Z$, and there is no edge from $M_{-}$to $Q_{-} \cup Q_{0}$. If $(Y \cup Z) \cap Q_{+}=\emptyset$, then $(Y \cup Z) \cap M_{-}$ must be empty as well, contradiction. If $(Y \cup Z) \cap Q_{+} \neq \emptyset$, apply statement iv) in Lemma 2:

$$
\omega_{(Y \cup Z) \cap Q_{+}}<p_{g\left((Y \cup Z) \cap Q_{+}\right) \cap M_{-}} \leqslant p_{(Y \cup Z) \cap M_{-}}
$$

where the second inequality follows from the fact that there is no edge between an agent in $X$ and a resource in $Y \cup Z$. This is the desired contradiction.

Substep 2.2. We focus now on $M_{+}$and $Q_{-}$, and show $M_{+}, Q_{-} \subseteq Y$, in particular, there is no green agent in $M_{+}$, and $x^{M_{+}} \geqslant p^{M_{+}}$.

Assume to the contrary $(X \cup Z) \cap M_{+} \neq \emptyset$. Then we have

$$
x_{(X \cup Z) \cap M_{+}} \leqslant p_{(X \cup Z) \cap M_{+}}<\omega_{f\left((X \cup Z) \cap M_{+}\right) \cap Q_{-}} \leqslant \omega_{(X \cup Z) \cap Q_{-}}
$$

where the first inequality is because the nodes in $(X \cup Z) \cap M_{+}$are green or black, the second is from statement ii) in Lemma 2, and the third because there is no edge from $X \cup Z$ to $Y$. Similarly we have

$$
x_{(X \cup Z) \cap M_{0}} \leqslant p_{(X \cup Z) \cap M_{0}} \leqslant \omega_{f\left((X \cup Z) \cap M_{0}\right) \cap Q_{0}} \leqslant \omega_{(X \cup Z) \cap Q_{0}}
$$

where the only differences are that the middle inequality, from statement iii) in Lemma 2, is not strict, and the fact that $(X \cup Z) \cap M_{0}$ could be empty. On the other hand

$$
x_{(X \cup Z) \cap\left(M_{+} \cup M_{0}\right)} \geqslant \omega_{(X \cup Z) \cap\left(Q_{-} \cup Q_{0}\right)}
$$

because the only edges in $G$ to $Q_{-} \cup Q_{0}$ are from $M_{+} \cup M_{0}$, and a resource in $X \cup Z$ can receive a positive flow only from one in $X \cup Z$.

Summing up inequalities (8), (9), (10), gives a contradiction. Hence $(X \cup Z) \cap M_{+}$must be empty after all, i.e., $M_{+} \subseteq Y$. Now (10) becomes $x_{(X \cup Z) \cap M_{0}} \geqslant \omega_{(X \cup Z) \cap\left(Q_{-} \cup Q_{0}\right)}$, whereas (9) gives $x_{(X \cup Z) \cap M_{0}} \leqslant \omega_{(X \cup Z) \cap Q_{0}}$. Whether $(X \cup Z) \cap M_{0}$ is empty or not, this implies $\omega_{(X \cup Z) \cap Q_{-}}=0$, i.e. $Q_{-} \subseteq Y$ as announced.

We derive a few more facts. First, all inequalities in (9) must be equalities, therefore $x^{(X \cup Z) \cap M_{0}}=p^{(X \cup Z) \cap M_{0}}$. Second, a positive flow to $(X \cup Z) \cap Q_{0}$ cannot come from $M_{-}$(statement i) in Lemma 2), or from $M_{+}$(because $\left.M_{+} \subset Y\right)$, therefore it only comes from $(X \cup Z) \cap M_{0}$.

\footnotetext{
5 When we speak below of a path, we always mean a directed path.
} 


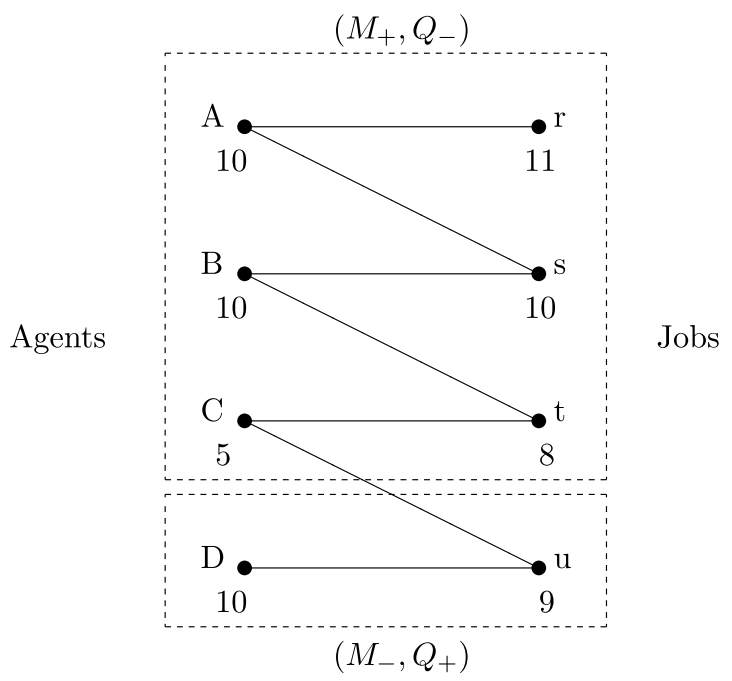

Fig. 3. Gallai-Edmonds decomposition and Pareto set I.

We showed in the previous paragraph $x_{(X \cup Z) \cap M_{0}}=\omega_{(X \cup Z) \cap Q_{0}}$. Therefore the entire flow from $(X \cup Z) \cap M_{0}$ goes to $(X \cup Z) \cap Q_{0}$.

Substep 2.3. We focus finally on $(Y \cup Z) \cap M_{0}$. The flow from $(Y \cup Z) \cap M_{0}$ cannot go to $Q_{-}$ $\left(G\left(M_{0}, Q_{-}\right)=\emptyset\right)$, or to $Q_{+}($contained in $X)$, or to $X \cap M_{0}$, so it must end up in $(Y \cup Z) \cap Q_{0}$. Together with the last statement in Step 2.2, this shows that the entire flow from $M_{0}$ goes to $Q_{0}$.

Assume for a moment that on $M_{0}$ we have $x^{M_{0}}=p^{M_{0}}$ : because $\left(G\left(M_{0}, Q_{0}\right), \omega, p\right)$ is balanced, this implies that there is no other flow coming to $Q_{0}$, in particular there is no flow on $G\left(M_{+}, Q_{0}\right)$, or $G\left(M_{0}, Q_{+}\right)$. Finally, as $Q_{+} \subseteq X$ and $M_{+} \subseteq Y$, there is no flow on $G\left(M_{+}, Q_{+}\right)$ either, which completes the proof of statements iii) and iv).

It remains to show $x^{(Y \cup Z) \cap M_{0}}=p^{(Y \cup Z) \cap M_{0}}$ (we already know this is true on $(X \cup Z) \cap M_{0}$ from Step 2.2). We have

$$
p_{(Y \cup Z) \cap M_{0}} \leqslant x_{(Y \cup Z) \cap M_{0}} \leqslant \omega_{(Y \cup Z) \cap Q_{0}}
$$

The first inequality because the nodes in $Y \cup Z$ are red or black, the second one because we saw above that the flow from $(Y \cup Z) \cap M_{0}$ goes to $(Y \cup Z) \cap Q_{0}$. Next we have

$$
\omega_{(Y \cup Z) \cap Q_{0}} \leqslant p_{g\left((Y \cup Z) \cap Q_{0}\right) \cap M_{0}} \leqslant p_{(Y \cup Z) \cap M_{0}}
$$

the first one because $\left(G\left(M_{0}, Q_{0}\right), \omega, p\right)$ is balanced, the second one because there is no edge from $X \cap M_{0}$ to $Y \cup Z$. Together, (11) and (12) imply $x^{(Y \cup Z) \cap M_{0}}=p^{(Y \cup Z) \cap M_{0}}$, as desired.

We illustrate Proposition 1 by two examples, each with four agents and four resources.

In Fig. 3 , the peaks are $p=(10,10,5,10)$ and the resources $\omega=(11,10,8,9)$. The two dashed-line boxes show the GE decomposition: resources $r, s, t$ are underdemanded by $A, B, C$, $u$ is overdemanded by $D\left(\left(M_{0}, Q_{0}\right)\right.$ is absent). Most of the inequalities in the system (4), (5), (6) are redundant, and the Pareto optimal set is given by

$$
\begin{aligned}
& x_{A}+x_{B}+x_{C}=29, \quad x_{D}=9 \\
& x_{A} \geqslant 11, \quad x_{B} \geqslant 10, \quad x_{C} \geqslant 5
\end{aligned}
$$




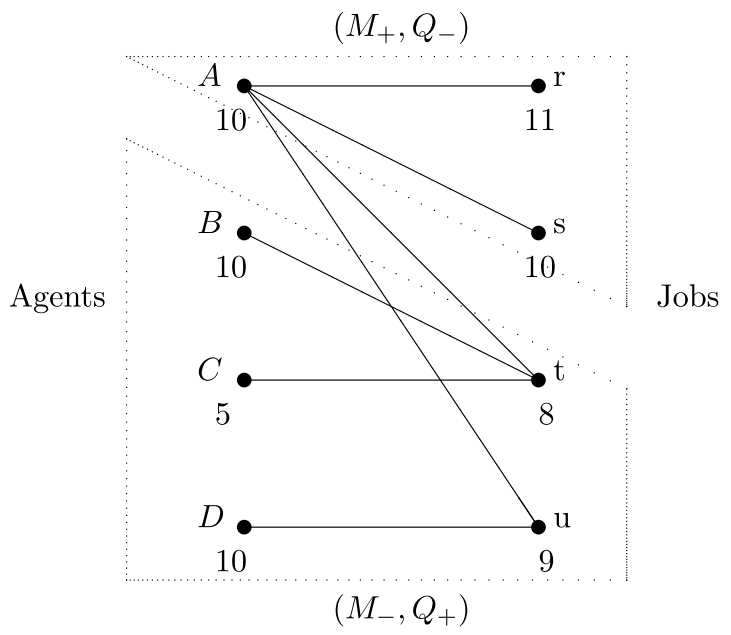

Fig. 4. Gallai-Edmonds decomposition and Pareto set II.

In Fig. 4, we use the same profile of peaks and resources as in the previous example, but the feasibility constraints have changed. The GE decomposition has now resources $r, s$ underdemanded by $A$, while $t, u$ are overdemanded by $B, C, D\left(\left(M_{0}, Q_{0}\right)\right.$ is absent). Note that the graph $G\left(M_{-}, Q_{+}\right)$is disconnected. The system (4), (5), (6) reduces to

$$
x_{A}=21, \quad x_{B}+x_{C}=8, \quad x_{C} \leqslant 5, \quad x_{D}=9
$$

\section{The Egalitarian solution}

There are two ways to define the Egalitarian solution, one algorithmic, the other as the Lorenz dominant element Pareto optimal allocation: Proposition 2 below shows the equivalence of these two definitions. We start by the algorithmic definition, using an ascending algorithm for the $M_{+}$ coordinates of the solution, and a descending one for the $M_{-}$coordinates. ${ }^{6}$

Given a problem $(G, \omega, p)$, we write our solution as $\mathcal{E}(G, \omega, p)$. By Pareto Optimality (5), $\mathcal{E}(G, \omega, p)^{M_{+}}$satisfies (4), $\mathcal{E}(G, \omega, p)^{M_{-}}$satisfies (6), and $\mathcal{E}(G, \omega, p)^{M_{0}}=p^{M_{0}}$.

The notation $Z^{1,2, \ldots, k}=\bigcup_{1}^{k} Z^{l}$ is used in the definition below and beyond.

Computing $\mathcal{E}(\boldsymbol{G}, \boldsymbol{\omega}, \boldsymbol{p})^{\boldsymbol{M}_{+}}$. We use an ascending algorithm based on the following system $\Theta(\lambda)$ of inequalities, where $\lambda$ is a nonnegative parameter:

$$
\Theta(\lambda): \quad \gamma_{S}(\lambda) \leqslant \omega_{f}(S) \cap Q_{-} \quad \text { for all } S \subseteq M_{+}
$$

where for all $i \in M_{+}, \gamma_{i}(\lambda)=\max \left\{\lambda, p_{i}\right\}$, so that $p^{M_{+}} \leqslant \gamma(\lambda)$ for all $\lambda$.

For $\lambda=0, \gamma(0)=p^{M_{+}}$and $\Theta(0)$ holds true, even strictly, because there is underdemand in $\left(G\left(M_{+}, Q_{-}\right), \omega, p\right)$ (Lemma 2). For $\lambda=\infty, \gamma(\infty)=\infty$. Therefore there is a smallest number $\lambda^{1}$, strictly positive, such that one of the inequalities in $\Theta\left(\lambda^{1}\right)$ is tight. As $S \rightarrow \omega_{f}(S) \cap Q_{-}-$ $\gamma_{S}\left(\lambda^{1}\right)$ is submodular, if two different subsets of $M_{+}$satisfy the equality $\gamma_{S}\left(\lambda^{1}\right)=\omega_{f(S) \cap Q_{-}}$

\footnotetext{
6 Chandramouli and Sethuraman [6] propose a single algorithm to define the Egalitarian solution, but the proof of Proposition 2 is more transparent with our two-pronged algorithm.
} 
then so do their union and intersection. We call $S^{1}$ the largest such subset. By statement ii) in Lemma 1 applied to $G\left(S^{1}, f\left(S^{1}\right) \cap Q_{-}\right)$, the (restricted) allocation $\gamma_{i}\left(\lambda^{1}\right)$ for the agents in $S^{1}$ is feasible by using all the resources in $f\left(S^{1}\right) \cap Q_{-}$and no more.

In the restricted problem $\left(G\left(M_{+} \backslash S^{1}, Q_{-} \backslash f\left(S^{1}\right)\right), \omega\right)$ the bilateral graph is described by $f^{1}(S)=\left(f(S) \backslash f\left(S^{1}\right)\right) \cap Q_{-}$. We claim $\gamma_{S}\left(\lambda^{1}\right)<\omega_{f^{1}(S)}$ for all nonempty $S \subseteq M_{+} \backslash S^{1}$. Indeed $\Theta\left(\lambda^{1}\right)$ is true and $S^{1}$ is the largest set such that the corresponding inequality is tight, therefore $\gamma_{S \cup S^{1}}\left(\lambda^{1}\right)<\omega_{f\left(S \cup S^{1}\right) \cap Q_{-}} \Leftrightarrow \gamma_{S}\left(\lambda^{1}\right)+\gamma_{S^{1}}\left(\lambda^{1}\right)<\omega_{f^{1}(S)}+\omega_{f\left(S^{1}\right) \cap Q_{-}}$.

Repeating the argument above, there is a smallest number $\lambda^{2}$, strictly above $\lambda^{1}$, at which one of the inequalities $\gamma_{S}(\lambda)<\omega_{f^{1}(S)}, S \subseteq M_{+} \backslash S^{1}$, becomes an equality. We call $S^{2}$ the largest such subset of $M_{+} \backslash S^{1}$. The allocation $\gamma_{i}\left(\lambda^{2}\right)$ for the agents in $S^{2}$ is achievable by using precisely all the resources in $f\left(S^{2}\right) \backslash f\left(S^{1}\right)$ (Lemma 1$)$.

Continuing in this fashion, we obtain a partition $S^{1}, S^{2}, \ldots, S^{K}$, of $M_{+}$, and a strictly increasing sequence $\lambda^{1}<\lambda^{2}<\cdots<\lambda^{K}$, such that for all $k, 1 \leqslant k \leqslant K$, the allocation $\gamma_{i}\left(\lambda^{k}\right)$ to the agents in $S^{k}$ is feasible by assigning the resources in $f\left(S^{k}\right) \backslash f\left(S^{1, \ldots, k-1}\right)$ to these agents. By construction this allocation is bounded below by $p^{M_{+}}$.

Computing $\mathcal{E}(\boldsymbol{G}, \omega, \boldsymbol{p})^{\boldsymbol{M}_{-}}$. Turning to the agents in $M_{-}$, we use a descending algorithm based on the system $\Xi(\mu)$ with nonnegative parameter $\mu$ :

$$
\Xi(\mu): \quad \omega_{T} \leqslant \delta_{g(T) \cap M_{-}}(\mu) \text { for all } T \subseteq Q_{+}
$$

where for all $i \in M_{-}, \delta_{i}(\mu)=\min \left\{\mu, p_{i}\right\}$, so that $\delta(\mu) \leqslant p^{M_{-}}$for all $\mu$.

We have $\delta(\infty)=p^{M_{-}}$, so $\Xi(\infty)$ is true, even strictly, because there is overdemand in $\left(G\left(M_{-}, Q_{+}\right), \omega, p\right)$ (Lemma 2). Also, $\delta(0)=0$, therefore there is a largest number $\mu^{1}$ such that one of the inequalities in $\Xi\left(\mu^{1}\right)$ is tight. We let $T^{1}$ be the largest subset of $Q_{+}$for which we have an equality (its existence guaranteed by the submodularity of $\left.T \rightarrow \delta_{g(T) \cap M_{-}}\left(\mu^{1}\right)-\omega_{T}\right)$. The allocation $\delta_{i}\left(\mu^{1}\right)$ to the agents of $g\left(T^{1}\right) \cap M_{-}$is feasible by using exactly the resources in $T^{1}$ (statement iii) in Lemma 1 applied to $G\left(g\left(T^{1}\right) \cap M_{-}, T^{1}\right)$ ). We repeat this construction in the restricted problem $\left(G\left(M_{-} \backslash g\left(T^{1}\right), Q_{+} \backslash T^{1}\right), \omega, p\right)$, etc.

We end up with a partition $T^{1}, \ldots, T^{L}$ of $Q_{+}$, and a strictly decreasing sequence $\mu^{1}>$ $\cdots>\mu^{L}$, such that for all $l, 1 \leqslant l \leqslant L$, the allocation $\delta_{i}\left(\mu^{l}\right)$ to the agents in $g\left(T^{l}\right) \backslash g\left(T^{1, \ldots, l-1}\right)$ is feasible by assigning exactly the resources in $T^{l}$ to these agents. By construction this allocation is bounded above by $p^{M_{-}}$. This concludes the definition of $\mathcal{E}(G, \omega, p)$.

By Proposition $1, \mathcal{E}(G, \omega, p)$ is Pareto Optimal. It is also Peak-Only.

The main normative property is Lorenz dominance. For any finite set $N$ and any $z \in \mathbb{R}^{N}$, denote by $z^{*}$ the order statistics of $z$, obtained by rearranging the coordinates of $z$ in increasing order: $z^{* 1} \leqslant z^{* 2} \leqslant \cdots \leqslant z^{* n}$. Say that $z$ Lorenz dominates $w$, written $z L D w$, if for all $k, 1 \leqslant$ $k \leqslant n$

$$
\sum_{t=1}^{k} z^{* t} \geqslant \sum_{t=1}^{k} w^{* t}
$$

We say that $z$ obtains from $w$ by a Pigou-Dalton transfer if there exist $i, j, 1 \leqslant i<j \leqslant n$, such that $z_{k}=w_{k}$ for all $k \neq i, j$, and

$$
w_{i}<z_{i} \leqslant z_{j}<w_{j} \quad \text { and } \quad z_{i}+z_{j}=w_{i}+w_{j}
$$

In this case $z$ Lorenz dominates $w$, strictly. This fact is used in the proofs of Proposition 3 and Theorem 1. 
Finally we say that $z$ is Lorenz dominant in the set $A$ if $z L D z^{\prime}$ for all $z^{\prime} \in A$. The relation $L$ is a partial ordering: not every set, even convex and compact, admits a Lorenz dominant element. On the other hand, in a convex set $A$ there can be at most one Lorenz dominant element.

Proposition 2. For any economy $(G, \omega, R)$ with peaks $p$, the Egalitarian solution $\mathcal{E}(G, \omega, p)$ is the Lorenz dominant Pareto optimal allocation:

$$
\mathcal{E}(G, \omega, p) L D x \quad \text { for all } x \in \mathcal{P O}(G, \omega, p)
$$

Proof. We set $x=\mathcal{E}(G, \omega, p)$. By Proposition 1, we need to prove two statements: $x^{M_{+}}$ (resp. $\left.x^{M_{-}}\right)$is Lorenz dominant within $\mathcal{A}\left(G\left(M_{+}, Q_{-}\right), \omega\right) \cap\left\{y \in \mathbb{R}_{+}^{M_{+}} \mid y \geqslant p^{M_{+}}\right\}$(resp. $\left.\mathcal{A}\left(G\left(M_{-}, Q_{+}\right), \omega\right) \cap\left\{y \in \mathbb{R}_{+}^{M_{-}} \mid y \leqslant p^{M_{-}}\right\}\right)$. We prove each statement in turn.

Step 1. We show that $x^{M_{+}}$is Lorenz dominant in $\mathcal{A}\left(G\left(M_{+}, Q_{-}\right), \omega\right) \cap\left\{y \in \mathbb{R}_{+}^{M_{+}} \mid y \geqslant p^{M_{+}}\right\}$.

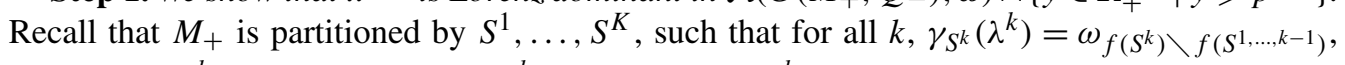
and for $i \in S^{k}$ we have $x_{i}=\max \left\{\lambda^{k}, p_{i}\right\}$. Moreover $\lambda^{k}$ is strictly increasing in $k$. We further partition $S^{k}$ as follows

$$
A^{k}=\left\{i \in S^{k} \mid x_{i}=\lambda^{k}>p_{i}\right\} ; \quad B^{k}=\left\{i \in S^{k} \mid x_{i}=p_{i} \geqslant \lambda^{k}\right\}
$$

We check first that $A^{k}$ is nonempty for all $k$. By Lemma 2 ii)

$$
p_{S^{1}}<\omega_{f\left(S^{1}\right)}=\sum_{i \in S^{1}} \max \left\{\lambda^{1}, p_{i}\right\}
$$

so $A^{1}$ is nonempty. Next

$$
p_{S^{2}} \leqslant \gamma_{S^{2}}\left(\lambda^{1}\right)<\omega_{f\left(S^{2}\right) \backslash f\left(S^{1}\right)}=\sum_{i \in S^{2}} \max \left\{\lambda^{2}, p_{i}\right\}
$$

where the strict inequality is explained in the construction of $\mathcal{E}(G, \omega, p)$. So $A^{2}$ is nonempty. And so on by induction on $k$.

The key to the proof is to choose a particular labeling of the agents in $M_{+}$as $\left\{1,2, \ldots, m_{+}\right\}$ such that the sequence $x_{i}$ is weakly increasing in $i$. We define

$$
\widetilde{B}^{k}=\left\{i \in B^{1, \ldots, k} \mid \lambda^{k} \leqslant x_{i}<\lambda^{k+1}\right\} \quad \text { for all } k, 1 \leqslant k \leqslant K
$$

with the convention $\lambda^{K+1}=\infty$.

Then the first $\left|A^{1}\right|$ coordinates of $x^{M_{+}}$are those of $A^{1}$ (they are all equal to $\lambda^{1}$ ), the next $\left|\widetilde{B}^{1}\right|$ ones are those of $\widetilde{B}^{1}$ in increasing order, followed by those of $A^{2}$, those of $\widetilde{B}^{2}$ in increasing order, and so on. Clearly $\widetilde{B}^{1, \ldots, K}=B^{1, \ldots, K}$ because if $x_{i}=p_{i} \in\left[\lambda^{k}, \lambda^{k+1}\left[\right.\right.$, then $i$ is in some $B^{k^{\prime}}$ for $k^{\prime} \leqslant k$.

We fix now $y \in \mathcal{A}\left(G\left(M_{+}, Q_{-}\right), \omega\right)$ such that $y \geqslant p^{M_{+}}$, and prove it is Lorenz dominated by $x^{M_{+}}$. We write $y^{*}(i)=\sum_{j=1}^{i} y^{* j}$ for the sum of the $i$ smallest coordinates of $y$, so that $y_{S} \geqslant y^{*}(|S|)$ for all $S \subseteq M_{+}$, and $\frac{y^{*}(i)}{i}$ increases weakly in $i$. As the coordinates of $x^{M_{+}}$are weakly increasing, we have $x_{\{1, \ldots, i\}}=x^{*}(i)$ for all $i$. We must prove $y^{*}(i) \leqslant x^{*}(i)$ for all $i$. We show by induction on $k=1, \ldots, K$, the property

$$
\mathcal{P}_{k}: \quad y^{*}(i) \leqslant x^{*}(i) \quad \text { for } 1 \leqslant i \leqslant\left|A^{1, \ldots, k}\right|+\left|\widetilde{B}^{1, \ldots, k}\right|
$$


We start with $\mathcal{P}_{1}$. By feasibility (Lemma 1) $y_{S^{1}} \leqslant \omega_{f\left(S^{1}\right)}=x_{S^{1}}$. On the other hand $y \geqslant p^{M_{+}}$ implies $y^{B^{1}} \geqslant x^{B^{1}}$ (as well as $y^{B^{1, \ldots, K}} \geqslant x^{B^{1, \ldots, K}}$ ). As $S^{1}=A^{1} \cup B^{1}$, the latter inequalities imply $y^{S^{1} \backslash S} \geqslant x^{S^{1} \backslash S}$ for any $S$ such that $A^{1} \subseteq S \subseteq S^{1}$, therefore

$y_{S} \leqslant x_{S}$ for all $S$ such that $A^{1} \subseteq S \subseteq S^{1}$

Choosing $S=\{1, \ldots, i\}$ in (15), with $\left|A^{1}\right| \leqslant i \leqslant\left|A^{1}\right|+\left|\widetilde{B}^{1}\right|$, we deduce

$$
y^{*}(i) \leqslant y(S) \leqslant x_{S}=x^{*}(i)
$$

Next for $S=\{1, \ldots, i\}$ with $i \leqslant\left|A^{1}\right|$, the inequality $y^{*}(i) \leqslant x^{*}(i)$ follows from (15) applied to $A^{1}$, the fact that $\frac{y^{*}(i)}{i}$ increases weakly in $i$, and that all coordinates of $x^{M_{+}}$are equal in $A^{1}$ :

$$
\frac{y^{*}(i)}{i} \leqslant \frac{y^{*}\left(\left|A^{1}\right|\right)}{\left|A^{1}\right|} \leqslant \frac{y_{A^{1}}}{\left|A^{1}\right|} \leqslant \frac{x_{A^{1}}}{\left|A^{1}\right|}=\frac{x^{*}(i)}{i}
$$

Thus $\mathcal{P}_{1}$ holds true. Turning to $\mathcal{P}_{2}$, we note that feasibility implies $y_{S^{1} \cup S^{2}} \leqslant \omega_{f\left(S^{1} \cup S^{2}\right)}=x_{S^{1} \cup S^{2}}$, and on the other hand $y^{B^{1} \cup B^{2}} \geqslant x^{B^{1} \cup B^{2}}$; from $S^{1,2}=A^{1,2} \cup B^{1,2}$ we deduce as above

$$
y_{S} \leqslant x_{S} \quad \text { for all } S \text { such that } A^{1,2} \subseteq S \subseteq S^{1,2}
$$

If $S=\{1, \ldots, i\}$ with $\left|A^{1,2}\right|+\left|\widetilde{B}^{1}\right| \leqslant i \leqslant\left|A^{1,2}\right|+\left|\widetilde{B}^{1,2}\right|$, the same inequalities (16) imply $y^{*}(i) \leqslant x^{*}(i)$. It remains to check the latter for $\left|A^{1}\right|+\left|\widetilde{B}^{1}\right| \leqslant i \leqslant\left|A^{1,2}\right|+\left|\widetilde{B}^{1}\right|$.

We set $a=i-\left(\left|A^{1}\right|+\left|\widetilde{B}^{1}\right|\right)$, and check first the inequality

$$
y^{*}(i) \leqslant y_{A^{1} \cup \widetilde{B}^{1}}+\frac{a}{\left|A^{2}\right|} y_{A^{2}}
$$

Setting $z=y^{A^{2}}$, we have $\frac{z^{*}(a)}{a} \leqslant \frac{z_{A^{2}}}{\left|A^{2}\right|}=\frac{y_{A^{2}}}{\left|A^{2}\right|}$ (by construction $a \leqslant\left|A^{2}\right|$ ). Thus $\frac{a}{\left|A^{2}\right|} y_{A^{2}}$ is not smaller than the $a$ smallest coordinates of $y$ in $A^{2}$. Inequality (18) follows.

Because all coordinates of $x^{M_{+}}$are equal in $A^{2}$, we have

$$
x^{*}(i)=x_{A^{1} \cup \widetilde{B}^{1}}+\frac{a}{\left|A^{2}\right|} x_{A^{2}}=\left(1-\frac{a}{\left|A^{2}\right|}\right) x_{A^{1} \cup \widetilde{B}^{1}}+\frac{a}{\left|A^{2}\right|} x_{A^{1,2} \cup \widetilde{B}^{1}}
$$

We rewrite inequality (18) as

$$
y^{*}(i) \leqslant y_{A^{1} \cup \widetilde{B}^{1}}+\frac{a}{\left|A^{2}\right|} y_{A^{2}}=\left(1-\frac{a}{\left|A^{2}\right|}\right) y_{A^{1} \cup \widetilde{B}^{1}}+\frac{a}{\left|A^{2}\right|} y_{A^{1,2} \cup \widetilde{B}^{1}}
$$

and note that inequality $y_{S} \leqslant x_{S}$ holds for $S=A^{1} \cup \widetilde{B}^{1}$ by (15), and for $S=A^{1,2} \cup \widetilde{B}^{1}$ by (17). The proof of $\mathcal{P}_{2}$ is complete.

The induction step from $\mathcal{P}_{k}$ to $\mathcal{P}_{k+1}$, proceeds in exactly the same way.

Step 2. We show that $x^{M_{-}}$is Lorenz dominant in $\mathcal{A}\left(G\left(M_{-}, Q_{+}\right), \omega\right) \cap\left\{y \in \mathbb{R}_{+}^{M_{-}} \mid y \leqslant p^{M_{-}}\right\}$. Recall that $Q_{+}$is partitioned as $T^{1}, \ldots, T^{L}$, such that the resources of $T^{\ell}$ are entirely assigned to agents in $S^{\ell}=g\left(T^{\ell}\right) \backslash g\left(T^{1, \ldots, \ell-1}\right)$, and $\omega_{T^{\ell}}=\delta_{S^{\ell}}\left(\mu^{\ell}\right)$ for all $k$, where $\mu^{\ell}$ is strictly decreasing; moreover $x_{i}=\min \left\{\mu^{\ell}, p_{i}\right\}$ for $i \in S^{\ell}$. As in Step 1 we partition $S^{\ell}$ as follows

$$
A^{\ell}=\left\{i \in S^{\ell} \mid x_{i}<p_{i} \Leftrightarrow \mu^{\ell}<p_{i}\right\} ; \quad B^{\ell}=\left\{i \in S^{\ell} \mid x_{i}=p_{i} \Leftrightarrow \mu^{\ell} \geqslant p_{i}\right\}
$$

The set $A^{1}$ is nonempty because $\sum_{i \in S^{1}} \min \left\{\mu^{1}, p_{i}\right\}=\omega_{T^{1}}<p_{S^{1}} ; A^{2}$ is nonempty because

$$
p_{S^{2}} \geqslant \delta_{S^{2}}\left(\mu^{1}\right)>\omega_{T^{2}}=\sum_{i \in S^{2}} \min \left\{\mu^{2}, p_{i}\right\}
$$


and so on by induction on $\ell$. As above the key is to choose a particular labeling of the agents in $M_{-}$as $\left\{1,2, \ldots, m_{-}\right\}$such that the sequence $x_{i}$ is weakly increasing in $i$. We define

$$
\widetilde{B}^{\ell}=\left\{i \in B^{1, \ldots, \ell} \mid \mu^{\ell} \geqslant x_{i}>\mu^{\ell+1}\right\} \text { for all } \ell, 1 \leqslant \ell \leqslant L
$$

with the convention $\mu^{L+1}=-\infty$.

Then in the reverse sequence $\left\{x_{m_{-}}, x_{m_{-}-1}, \ldots, x_{1}\right\}$ the first $\left|A^{1}\right|$ coordinates are those of $A^{1}$ (they are all equal to $\mu^{1}$ ), the next $\left|\widetilde{B}^{1}\right|$ ones are those of $\widetilde{B}^{1}$ in decreasing order, followed by those of $A^{2}$, those of $\widetilde{B}^{2}$ in decreasing order, and so on. Clearly $\widetilde{B}^{1, \ldots, L}=B^{1, \ldots, L}$ because if $\left.x_{i}=p_{i} \in\right] \mu^{\ell+1}, \mu^{\ell}$ ] then $i$ is in some $B^{\ell^{\prime}}$ for $\ell^{\prime} \leqslant \ell$.

For an arbitrary $y \in \mathcal{A}\left(G\left(M_{-}, Q_{+}\right), \omega\right)$ such that $y \leqslant p^{M_{-}}$, we write $y^{*}(i)=\sum_{j=m_{-} i+1}^{m_{-}} y^{* j}$ for the sum of the $i$ largest coordinates of $y$, so that $y_{S} \leqslant y^{*}(|S|)$ for all $S \subseteq M_{-}$. The end of the proof that $y$ is Lorenz dominated by $x^{M_{-}}$is entirely similar to the one in Step 1, upon reversing the direction of inequalities. That is, the feasibility constraints $\omega_{T} \leqslant y_{g(T)}$ imply now $y_{S^{1}} \geqslant \omega_{T^{1}}=x_{S^{1}}$; on the other hand $y^{B^{1}} \leqslant x^{B^{1}}$, and so (15) follows (up to a change of sign). Similarly the inequality $y_{S^{1,2}} \geqslant \omega_{T^{1,2}}=x_{S^{1,2}}$ holds by feasibility of $y$. We omit the details.

We illustrate the Egalitarian solution with a few examples.

No bilateral constraints. Here $G$ is the complete graph $G=M \times Q$. As mentioned already after Lemma 2, the model is equivalent to Sprumont's standard model. Suppose first we have overdemand, $\omega_{Q}<p_{M}$. Then $M=M_{-}, Q=Q_{+}$, and $g(T)=M$ for all $T$. Therefore system (14) reads

$$
\omega_{T} \leqslant \sum_{i \in M} \min \left\{\mu, p_{i}\right\} \quad \text { for all } T
$$

When $\mu$ is the largest number such that this is an equality for some $T$, it must be an equality for $Q$, therefore $\mu^{1}$ solves $\sum_{i \in M} \min \left\{\mu, p_{i}\right\}=\omega_{Q}$ and the algorithm stops after one step.

When the resources are underdemanded, $\omega_{Q}>p_{M}$, the algorithm stops similarly in one step: the number $\lambda^{1}$ solves $\sum_{i \in M} \max \left\{\lambda, p_{i}\right\}=\omega_{Q}$, and $S^{1}=M$.

Want all or nothing. In the standard model, the Egalitarian solution when all peaks are zero (resources are all "bad"), is the same as when all are infinite (resources are all "good"), namely it divides the resource equally. This is still true in the bipartite model. By Proposition 1, when $p=0$ we have $M=M_{+}, Q=Q_{-}$, and $\mathcal{P} \mathcal{O}(G, \omega, 0)=\mathcal{A}(G, \omega)$. Similarly $M=M_{-}, Q=Q_{+}$ when $p=\infty$, and $\mathcal{P} \mathcal{O}(G, \omega, \infty)=\mathcal{A}(G, \omega)$. By Proposition 2, the Egalitarian solution picks the Lorenz dominant feasible allocation in both problems. Note that it is also the Dutta-Ray Egalitarian solution [11] of the supermodular game $(M, w)$, and of its dual game $(M, v)$.

In the example of Fig. 2, Pareto optimal allocations assign the 14 units of overdemanded resources $t, u$ to agents $C, D$, and the 28 units of underdemanded $r, s$ to $A, B$. The Egalitarian solution is $x_{A}=13, x_{B}=15, x_{C}=9, x_{D}=5$.

In the example of Fig. 3, Pareto optimal allocations distribute 29 units to $A, B, C$, with peaks $10,10,5$ and the Egalitarian solution is $x_{A}=11, x_{B}=10, x_{C}=8$; and $x_{D}=9$.

In the example of Fig. 4 , we need to divide 8 units between $B, C$. Full equality $x_{B}=x_{C}=4$ is feasible and stays below both peaks, so $B$ and $C$ receive 4 in the Egalitarian solution. 


\section{The Egalitarian rule and its properties}

Definition. Given $M$ and $Q$, a rule $\psi$ selects for every economy $(G, \omega, R)$ a feasible allocation $\psi(G, \omega, R) \in \mathcal{A}(G, \omega)$. The Egalitarian rule $\mathcal{E}$ selects the Egalitarian solution $\mathcal{E}(G, \omega, p)$ for each $R \in \mathcal{R}^{M}$ with peak profile $p \in \mathbb{R}_{+}^{M}$.

Whenever $G$ and $\omega$ are fixed and this causes no confusion, we simply write a rule $\psi(G, \omega, R)$ as $\psi(R)$. In particular the definitions of properties are written for a generic solution $\psi$ and fixed $G$ as well as $\omega$.

We start with the generalization of Equal Treatment of Equals. There are two ways to state this requirement. The first one is welfarist: equal agents should be indifferent between their respective shares. The other one is physical: equal agents should receive the same share. Our generalization of Equal Treatment of Equals is in the physical form.

In our model agents differ in their preferences and in their feasibility constraints, so they are fully "equals" only when they share both characteristics. An egalitarian social planner concerned with efficiency will surely not treat equally two agents with different preferences, but, as noted in the introduction, he may or may not make them accountable for the differences in their connections. If he does not, as we assume in this paper, he will strive to compensate as much as possible for these ethically irrelevant differences. ${ }^{7}$ In particular he will try to give identical shares to any two agents with identical preferences, or at least to make their shares as equal as the feasibility constraints permit. Thus, ultimately, the difference in the shares of two agents with identical preferences comes from the difference in their respective feasibility constraints, but only because the latter precludes full equality of shares.

Consider the following example: two agents Ann (A) and Bob (B) with identical preferences peaking at 4 , and two resources with 2 units each: $\omega_{1}=\omega_{2}=2$. Ann can consume both resources, $f(A)=\{1,2\}$, while Bob can only consume $\omega_{2}, f(B)=\{2\}$. It is possible to give 2 units to Ann and 2 units to Bob (Ann gets $\omega_{1}$, and Bob gets $\omega_{2}$ ) thus ignoring the fact that Ann is better connected than Bob. If $\omega_{1}=1, \omega_{2}=2$, we would similarly give 1.5 units to each. But if $\omega_{1}=2, \omega_{2}=1$, the best we can do for Bob is to give him 1 unit $\left(\omega_{2}\right)$ while Ann gets 2 units $\left(\omega_{1}\right) .{ }^{8}$ We now state our generalizations of Equal Treatment of Equals and No Envy. We then come back to the above example to illustrate in turn these two properties.

Equal Treatment of Equal Demands. For each $R \in \mathcal{R}^{M}$ and $\{i, j\} \subseteq M$ such that $R_{i}=R_{j}$, if $\psi_{j}(R) \neq \psi_{i}(R)$ there exists no $x \in \mathcal{A}(G, \omega)$ such that

$$
\psi_{k}(R)=x_{k} \quad \text { for each } k \neq i, j \quad \text { and } \quad\left|x_{i}-x_{j}\right|<\left|\psi_{i}(R)-\psi_{j}(R)\right|
$$

In a classic fair division problem, the shares $x_{i}, x_{j}$ of two agents $i$ and $j$ can always be reallocated between these two, without affecting other agents' shares. In our model such a redistribution may not be feasible, or it may require to alter the allocation of agents other than $i$ and $j$. The axiom says that equalizing transfers among agents with identical preferences are legitimate only if they are feasible, and do not disrupt other agents' allocations.

\footnotetext{
7 Modern theories of distributive justice (e.g., Roemer [20], Fleurbaey [9]) emphasize the distinction between individual characteristics for which we are or are not held responsible, e.g., effort versus innate talent. A just distribution rule should reward more the most hardworking agent, but avoid penalizing the less talented.

8 If agents are held responsible for their connections, a consistent division rule gives all $\omega_{1}$ to Ann, then uses a standard method to divide $\omega_{1}$ between Bob with a claim of 4 and Ann with a residual claim of $(4-2)$. See [18].
} 
Turning to the familiar test of No Envy, we postulate similarly that agent $i$ 's envy of agent $j$ 's allocation is legitimate only if it is feasible to improve upon agent $i$ 's allocation without altering the allocation of anyone other than agent $j$.

No Envy. For each $R \in \mathcal{R}^{M}$ and $\{i, j\} \subseteq M$ such that $\psi_{j}(R) P_{i} \psi_{i}(R)$, there exists no $x \in$ $\mathcal{A}(G, \omega)$ such that

$$
\psi_{k}(R)=x_{k} \quad \text { for each } k \neq i, j \quad \text { and } \quad x_{i} P_{i} \psi_{i}(R)
$$

We now go back to the preceding example to illustrate the two axioms. We show that each one of Equal Treatment of Equal Demands or No Envy may (or may not) force the Egalitarian solution for three possible resource endowments.

Modify the example above as follows: keep the resources constant at $\left(\omega_{1}, \omega_{2}\right)=(2,2)$, and add three agents $C, D, E$, all with preferences identical to $A$ and $B$ (in particular peak at 4 ). The pattern of compatibility is: $f(A)=\{1,2\}, f(B)=\{2\}, f(C)=f(D)=f(E)=\{1\}$. Equal Treatment of Equal Demands forces the allocation $\left(x_{A}, x_{B}, x_{C}, x_{D}, x_{E}\right)=\left(1,1, \frac{2}{3}, \frac{2}{3}, \frac{2}{3}\right)$ where $A$ and $B$ share $\omega_{2}$ while $C, D, E$ share $\omega_{1}$.

On the other hand, suppose that $C, D, E$ 's peaks are each 2 instead of 4 , with no other changes in the problem. Then Equal Treatment of Equal Demands is satisfied by the allocations $(\alpha, \alpha, \beta, \beta, \beta)$, where $0 \leqslant \beta \leqslant \frac{2}{3}, 1 \leqslant \alpha \leqslant 2,2 \alpha+3 \beta=4$, and only those. But No Envy singles out the Egalitarian solution $\left(1,1, \frac{2}{3}, \frac{2}{3}, \frac{2}{3}\right)$.

This example shows that Equal Treatment of Equal Demands does not by itself select the Lorenz dominant Pareto optimal allocation.

Our final illustration is one where No Envy does not single out this allocation either. Consider the problem of Fig. 3. There the Egalitarian allocation is $(11,10,8,9)$. Note that $A$ prefers the share of $B$ but no feasible allocation allows a lower load for $A$, so No Envy holds. Consider the Pareto optimal allocation $\left(x_{A}, x_{B}, x_{C}, x_{D}\right)=(11,11,7,9)$, where each of $A, B, C$ bears a piece of the excess supply. If $11 P_{B} 7$ this allocation meets No Envy. If $7 P_{B} 11$ (recall that the peak of $R_{B}$ is at 10$), B$ envies $C$, and this is legitimate because $(11,10,8,9)$ is feasible and improves upon $B$ while affecting only $C$.

In the standard model, No Envy implies the welfarist Equal Treatment of Equals, and No Envy coupled with Pareto Optimality implies the physical Equal Treatment of Equals. Only the latter implication is true in our model.

\section{Proposition 3. Fix $G$ and $\omega$.}

i) Pareto Optimality and No Envy imply Equal Treatment of Equal Demands.

ii) The Egalitarian rule $\mathcal{E}$ satisfies No Envy.

Proof. Statement $i$ ). Suppose the rule $\psi$ violates Equal Treatment of Equal Demands and check it violates either No Envy or Pareto Optimality. Fix a profile $R$, an allocation $x$, and two agents 1,2 as in the premises of (20). Write $y=\psi(R)$ and assume without loss of generality $y_{1}<y_{2}$. Distinguish two cases. If 1 and 2's common peak $p$ is in $] y_{1}, y_{2}$ [, then for $\varepsilon$ small enough the allocation $z=(1-\varepsilon) y+\varepsilon x$, feasible by convexity of $\mathcal{A}(G, \omega)$, is Pareto superior to $y$. If $p \leqslant y_{1}<y_{2}$ $\left(y_{1}<y_{2} \leqslant p\right.$ ), then 2 envies 1 ( 1 envies 2$)$, and the allocation $x$ satisfies (21), contradiction. 
Statement ii). Fix a profile $R$ and set $y=\mathcal{E}(R)$. Assume 1 envies 2 at $y$ via the feasible allocation $x$. Recall that $x_{1}+x_{2}=y_{1}+y_{2}$, and $x_{k}=y_{k}$ for $k \geqslant 3$. No Envy at $y$ via $x$ means $y_{2} P_{1} y_{1}$ and $x_{1} P_{1} y_{1}$, so it precludes $y_{1}=p\left[R_{1}\right]$.

We now assume $y_{1}<p\left[R_{1}\right]$ and derive a contradiction. Agent 1 is in $M_{-}$by Proposition 1 , and single-peakedness of $R_{1}$ implies $y_{1}<y_{2}, x_{1}$. We claim that agent 2 must be in $M_{-}$as well: if he is not, it is feasible to increase the share of 1 at $y$ while keeping that of all other $M_{-}$agents constant, which cannot be because $M_{-}$can only consume $Q_{+}$, and is already eating all up at $y$ (Proposition 1). Thus $\{1,2\} \subseteq M_{-}$. Now we have $y_{1}<x_{1}, y_{2}>x_{2}$ (as $x_{1}+x_{2}=y_{1}+y_{2}$ ), and for $\varepsilon$ small enough the allocation $\varepsilon x+(1-\varepsilon) y$ is Pareto optimal by Proposition 1. Because $y_{1}<y_{2}$, it is also, for $\varepsilon$ small enough, a Pigou-Dalton transfer from 2 to 1 at $y$, contradicting the Lorenz dominance property of $y$.

When we assume $y_{1}>p\left[R_{1}\right]$, agent 1 is in $M_{+}$, and single-peakedness of $R_{1}$ implies $y_{1}>$ $y_{2}, x_{1}$. If agent 2 was not in $M_{+}$, it would be feasible to decrease the share of 1 at $y$ while keeping everyone else in $M_{+}$fixed. But $M_{+}$must consume all of $Q_{-}$in any feasible allocation, contradiction. From there the argument exactly parallels that in the previous case.

We turn to Strategyproofness, and go back for clarity to the notation $p\left[R_{i}\right]$ for the peak of $R_{i}$. We define two additional properties, already familiar in the standard model.

Monotonicity. For all $R \in \mathcal{R}^{M}, i \in M$, and $R_{i}^{\prime} \in \mathcal{R}^{i}$

$$
p\left[R_{i}^{\prime}\right] \leqslant p\left[R_{i}\right] \Rightarrow \psi_{i}\left(R_{i}^{\prime}, R_{-i}\right) \leqslant \psi_{i}(R)
$$

Invariance. ${ }^{9}$ For all $R \in \mathcal{R}^{M}, i \in M$, and $R_{i}^{\prime} \in \mathcal{R}^{i}$

$$
\begin{aligned}
& \left\{p\left[R_{i}\right]<\psi_{i}(R) \text { and } p\left[R_{i}^{\prime}\right] \leqslant \psi_{i}(R)\right\} \Rightarrow \psi_{i}\left(R_{i}^{\prime}, R_{-i}\right)=\psi_{i}(R) \\
& \left\{p\left[R_{i}\right]>\psi_{i}(R) \text { and } p\left[R_{i}^{\prime}\right] \geqslant \psi_{i}(R)\right\} \Rightarrow \psi_{i}\left(R_{i}^{\prime}, R_{-i}\right)=\psi_{i}(R)
\end{aligned}
$$

As in the standard model [7], Monotonicity implies own-peak-only, namely $p\left[R_{i}^{\prime}\right]=p\left[R_{i}\right] \Rightarrow$ $\psi_{i}\left(R_{i}^{\prime}, R_{-i}\right)=\psi_{i}(R)$ : my allocation depends only upon the peak of my preferences. Abusing notation, we can write $\psi_{i}\left(p_{i}, R_{-i}\right)$ instead of $\psi_{i}(R)$. Then Monotonicity and Invariance imply that the mapping $p_{i} \rightarrow \psi_{i}\left(p_{i}, R_{-i}\right)$ is continuous (because it is constant around any $p_{i}$ such that $\left.p_{i} \neq \psi_{i}\left(p_{i}, R_{-i}\right)\right)$.

Strategyproofness. For all $R \in \mathcal{R}^{M}, i \in M$, and $R_{i}^{\prime} \in \mathcal{R}^{i}$,

$$
\psi_{i}(R) R_{i} \psi_{i}\left(R_{i}^{\prime}, R_{-i}\right)
$$

The next lemma states two important connections between the three axioms above.

Lemma 3. Fix $G$ and $\omega$.

i) If a rule is Monotonic and Invariant, it is Strategyproof;

ii) A Pareto Optimal and Strategyproof rule is Monotonic and Invariant.

\footnotetext{
9 Invariance is sometimes called Uncompromisingness in the literature on Strategyproofness.
} 
Proof. Statement i) is proven just as in the standard model (see [7]).

Statement ii). Fix a Pareto Optimal and Strategyproof rule $\psi$. We show first that the mapping $R_{i} \rightarrow \psi_{i}\left(R_{i}, R_{-i}\right)$ is Peak-Only. Fix $R_{-i}$ and consider two preferences $R_{i}, R_{i}^{\prime}$ such that $p\left[R_{i}\right]=p\left[R_{i}^{\prime}\right]$. The GE decomposition (Lemma 2) is the same in $R$ and $\left(R_{i}^{\prime}, R_{-i}\right)$, so by Pareto Optimality agent $i$ 's allocations $x_{i}=\psi_{i}(R)$ and $x_{i}^{\prime}=\psi_{i}\left(R_{i}^{\prime}, R_{-i}\right)$ are on the same side of $p\left[R_{i}\right]$. Now Strategyproofness implies Peak-Only.

We show now that $\psi$ is Invariant. Under the premises of property (22), if $\psi_{i}\left(R_{i}^{\prime}, R_{-i}\right)>\psi_{i}(R)$ we have $p\left[R_{i}^{\prime}\right] \leqslant \psi_{i}(R)<\psi_{i}\left(R_{i}^{\prime}, R_{-i}\right)$, hence a violation of Strategyproofness for agent $i$ at $R_{i}^{\prime}$. If $\psi_{i}\left(R_{i}^{\prime}, R_{-i}\right)<\psi_{i}(R)$ we can find a preference $R_{i}^{*}$ such that $p\left[R_{i}^{*}\right]=p_{i}\left[R_{i}\right]$ and $\psi_{i}\left(R_{i}^{\prime}, R_{-i}\right) P_{i}^{*} \psi_{i}(R)$. By Peak-Only, $\psi_{i}\left(R_{i}^{*}, R_{-i}\right)=\psi_{i}(R)$, so agent $i$ with preferences $R_{i}^{*}$ benefits by reporting $R_{i}^{\prime}$. The proof of (23) is identical.

Next we prove Monotonicity. We fix $i, R$ and $R_{i}^{\prime}$ such that $p_{i}^{\prime}=p\left[R_{i}^{\prime}\right] \leqslant p\left[R_{i}\right]=p_{i}$, and let $p, p^{\prime}$ be the profile of peaks at $R$ and $\left(R_{i}^{\prime}, R_{-i}\right)$ respectively. Set as above $x_{i}=\psi_{i}(R), x_{i}^{\prime}=$ $\psi_{i}\left(R_{i}^{\prime}, R_{-i}\right)$. We use the notation $M_{-}(p)$ for the $M_{-}$component of the GE decomposition at $(G, \omega, p)$, and define similarly $M_{+}\left(p^{\prime}\right)$ etc.

We distinguish two cases.

Case 1: $i \in M_{-}(p)$. Assume first $p_{i}^{\prime}>x_{i}$. Then the decomposition is unchanged, in particular $M_{-}(p)=M_{-}\left(p^{\prime}\right)$, so by Pareto Optimality $x_{i}^{\prime} \leqslant p_{i}^{\prime}$. Assume $x_{i}<x_{i}^{\prime}$; then we have $x_{i}<x_{i}^{\prime} \leqslant$ $p_{i}^{\prime} \leqslant p_{i}$, and we get a contradiction of Strategyproofness for agent $i$ at profile $R$. Assume next $p_{i}^{\prime} \leqslant x_{i}$. Then $x_{i}<x_{i}^{\prime}$ would give $p_{i}^{\prime} \leqslant x_{i}<x_{i}^{\prime}$, a violation of Strategyproofness for agent $i$ with preference $R_{i}^{\prime}$.

Case 2: $i \in\left(M_{0} \cup M_{+}\right)(p)$. Then $p_{i} \leqslant x_{i}$, so $x_{i}<x_{i}^{\prime}$ would give $p_{i}^{\prime} \leqslant p_{i} \leqslant x_{i}<x_{i}^{\prime}$, hence a violation of Strategyproofness for agent $i$ at $R_{i}^{\prime}$.

Proposition 4. The Egalitarian rule is Monotonic and Invariant, hence Strategyproof as well.

Proof. We fix $(G, \omega, p)$, an agent $i$ and a benchmark profile of peaks $p$, with corresponding Egalitarian allocation $x$. We consider a change of peak by agent $i$ only, to $p_{i}^{\prime}$, and we write $p_{j}^{\prime}=p_{j}$ for all $j \neq i$, so that $p^{\prime}=\left(p_{i}^{\prime}, p_{-i}\right)$. We use the notation $M_{+}(p), M_{-}\left(p^{\prime}\right)$, etc., as in the previous proof. The long proof of Monotonicity is organized as follows.

Consider a shift $p_{i} \rightarrow p_{i}^{\prime}$ such that the GE decomposition does not change: by Lemma 2 this means $i \in M_{+}(p)=M_{+}\left(p^{\prime}\right)$ or $i \in M_{-}(p)=M_{-}\left(p^{\prime}\right)$ (if $i \in M_{0}(p)$ any shift destroys the budget-balance of $M_{0}(p)$ hence alters the decomposition). In Step 1 we prove Monotonicity of $\mathcal{E}$ for such a shift where $i \in M_{+}(p)=M_{+}\left(p^{\prime}\right)$. The decomposition does not change if $p_{i}^{\prime} \leqslant p_{i}$; we characterize in Step 2 the smallest report $p_{i}^{*}, p_{i}^{*}>p_{i}$, at which it does change, proving in particular that $i \in M_{0}\left(p^{*}\right)$. Symmetric results hold if we start from the situation $i \in M_{-}(p)$ (Step 3). It is then a simple matter to conclude in Step 4 by "concatenating" the moves from $p$ where $i \in M_{+}(p)$ to $p^{*}$ such that $i \in M_{0}\left(p^{*}\right)$, then from $p^{*}$ to $p^{\prime}$ such that $i \in M_{-}\left(p^{\prime}\right)$.

Step 1. We prove Monotonicity for a shift $p_{i} \rightarrow p_{i}^{\prime}$ such that $i \in M_{+}(p)=M_{+}\left(p^{\prime}\right)$.

Substep 1.a: assume $p_{i}^{\prime}<p_{i}$. We show $x_{i}^{\prime} \leqslant x_{i}$ by distinguishing two cases. Write in both cases $S^{k}, \lambda^{k}$ for the partition and corresponding parameters of the ascending algorithm at $p$, and let $i \in S^{\ell}, x_{i}=\lambda^{\ell} \vee p_{i}$.

Case 1: $p_{i}<\lambda^{\ell}=x_{i}$. Then the partition and corresponding parameters are unchanged at $p^{\prime}$ so that $x_{i}^{\prime}=x_{i}$.

Case 2: $p_{i}=x_{i} \geqslant \lambda^{\ell}$. Then $S^{k}, \lambda^{k}$ are unchanged for $1 \leqslant k \leqslant \ell-1$, but $S^{\ell}, \lambda^{\ell}$ may change. However for $\lambda=p_{i}$ we have 


$$
\sum_{j \in S^{\ell}} \lambda \vee p_{j}^{\prime} \geqslant \sum_{j \in S^{\ell}} \lambda^{\ell} \vee p_{j}^{\prime}=\omega_{T^{\ell}}
$$

where we set $T^{\ell}=f\left(S^{\ell}\right) \backslash f\left(S^{1, \ldots, \ell-1}\right)$. Therefore if $S^{\ell}$ changes, the new set $\tilde{S}^{\ell}$ contains $i$ and $\tilde{\lambda}^{\ell} \leqslant p_{i}$, hence $x_{i}^{\prime} \leqslant p_{i}=x_{i}$.

Substep 1.b: assume $p_{i}<p_{i}^{\prime}$. If $p_{i}^{\prime} \geqslant x_{i}$ notice that $i \in M_{+}\left(p^{\prime}\right)$ implies $x_{i}^{\prime} \geqslant p_{i}^{\prime}$ so we are done. We are left with the case $p_{i}<p_{i}^{\prime}<x_{i}=\lambda^{\ell}$, that requires more work.

We prove by induction on $\ell$ that the first $\ell$ pairs $\left(S^{k}, \lambda^{k}\right), 1 \leqslant k \leqslant \ell$, are unchanged at $p^{\prime}$. We write $\left(\widetilde{S}^{k}, \widetilde{\lambda}^{k}\right)$ for these pairs at $p^{\prime}$.

Suppose $\ell=1$, then $\sum_{j \in S} \lambda^{1} \vee p_{j}=\sum_{j \in S} \lambda^{1} \vee p_{j}^{\prime}$ for all $S \subseteq M_{+}(p)$, so the claim holds.

Next suppose $\ell \geqslant 2$. Assume $S^{1} \neq \widetilde{S}^{1}$ and derive a contradiction. This implies there exists $S \subseteq M_{+}(p), S$ a strict subset of $S^{1}$, such that

$$
\sum_{j \in S} \lambda^{1} \vee p_{j}^{\prime} \geqslant \omega_{f(S)}
$$

Indeed suppose (24) fails for all such sets $S$ : as $p$ and $p^{\prime}$ coincide inside $S^{1}$, we would get $S^{1}=\widetilde{S}^{1}$. Fix $S$ as in (24), that must contain $i$, hence $S \cap S^{\ell}$ is nonempty. By definition of the ascending algorithm, the sets $T^{1}=f\left(S \cap S^{1}\right), \ldots, T^{k}=f\left(S \cap S^{k}\right) \backslash\left(T^{1, \ldots, k-1}\right), \ldots$, are pairwise disjoint and $\sum_{S \cap S^{k}}^{k} \lambda^{k} \vee p_{j} \leqslant \omega_{T^{k}}$ for all $k$, therefore

$$
\sum_{1 \leqslant k \leqslant K}\left[\sum_{S \cap S^{k}} \lambda^{k} \vee p_{j}\right] \leqslant \omega_{f(S)}
$$

In view of (24), we get

$$
\sum_{1 \leqslant k \leqslant K}\left[\sum_{S \cap S^{k}} \lambda^{k} \vee p_{j}\right] \leqslant \sum_{j \in S} \lambda^{1} \vee p_{j}^{\prime}
$$

For all $k \neq \ell$, we have $\lambda^{k} \geqslant \lambda^{1}$ and $p^{S \cap S^{k}}=p^{\prime S \cap S^{k}}$, implying $\sum_{S \cap S^{k}} \lambda^{k} \vee p_{j} \geqslant \sum_{S \cap S^{k}} \lambda^{1} \vee p_{j}^{\prime}$. As $\lambda^{\ell}$ is larger than $\lambda^{1}, p_{i}^{\prime}$, and $p_{i}$, and $S \cap S^{\ell}$ is nonempty, we get $\sum_{S \cap S^{\ell}} \lambda^{\ell} \vee p_{j}>$ $\sum_{S \cap S^{\ell}} \lambda^{1} \vee p_{j}^{\prime}$. The desired contradiction follows and we conclude $S^{1}=\widetilde{S}^{1}$.

To show next $S^{2}=\widetilde{S}^{2}$, we replicate the above argument as follows. If $\ell=2$, then $\sum_{j \in S} \lambda^{2} \vee$ $p_{j}=\sum_{j \in S} \lambda^{2} \vee p_{j}^{\prime}$ for all $S \subseteq M_{+}(p) \backslash S^{1}$, because $p_{i}, p_{i}^{\prime}<\lambda^{2}$, and the claim holds. If $\ell \geqslant 3$ and $S^{2} \neq \widetilde{S}^{2}$, we can pick $S \subseteq M_{+}(p) \backslash S^{1}$ such that $S \subset S^{2}$ and

$$
\sum_{j \in S} \lambda^{2} \vee p_{j}^{\prime} \geqslant \omega_{f(S) \backslash S^{1}}
$$

and proceed as above by decomposing $S$ along $S^{k}, 2 \leqslant k \leqslant K$. The induction step is now clear.

Step 2. Starting from $i \in M_{+}(p)$ we study the critical value $p_{i}^{*}$, at which $i \in M_{+}\left(p^{\prime}\right)$ ceases to be true.

In the shift from $p$ to $p^{\prime}$, the subproblems $\left(G\left(M_{0}, Q_{0}\right), \omega, p\right)$ and $\left(G\left(M_{-}, Q_{+}\right), \omega, p\right)$ are unchanged, so by Lemma 2 the equality $M_{+}(p)=M_{+}\left(p^{\prime}\right)$ holds as long as $\left(G\left(M_{+}, Q_{-}\right), \omega, p^{\prime}\right)$ exhibits underdemand, i.e., as long as $p_{S}^{\prime}<\omega_{f(S) \cap Q_{-}(p)}$ for all $S \subseteq M_{+}(p)$. This is true in particular if $p_{i}^{\prime}<p_{i}$. Therefore the critical report $p_{i}^{*}$ at which the GE decomposition and the status of agent $i$ change is larger than $p_{i}$; it is the smallest number such that

$$
p_{S \backslash i}+p_{i}^{*}=\omega_{f(S) \cap Q_{-}(p)}
$$


for some subset $S$ of $M_{+}(p)$ containing $i$. Let $S^{*}$ be the largest $S$ satisfying (25) (well defined by the usual submodularity argument). Recall from the proof of Lemma 2 that $\left(M_{-} \cup M_{0}\right)(p)$ is the largest solution of $\arg \max _{S \subseteq M}\left\{p_{S}-\omega_{f(S)}\right\}$. Writing for brevity $\widetilde{S}=\left(M_{-} \cup M_{0}\right)(p)$, we claim that $\widetilde{S} \cup S^{*}$ is the largest solution of $\arg \max _{S \subseteq M}\left\{p_{S}^{*}-\omega_{f(S)}\right\}$, in other words $\widetilde{S} \cup S^{*}=$ $\left(M_{-} \cup M_{0}\right)\left(\underline{p}^{*}\right)$.

Recall $f(\widetilde{S})=\left(Q_{+} \cup Q_{0}\right)(p)$ and compute

$$
p_{\widetilde{S} \cup S^{*}}^{*}-\omega_{f\left(\widetilde{S} \cup S^{*}\right)}=\left(p_{\widetilde{S}}-\omega_{f(\widetilde{S})}\right)+\left(p_{S^{*}}^{*}-\omega_{f\left(S^{*}\right) \cap Q_{-}(p)}\right)=p_{\widetilde{S}}-\omega_{f(\widetilde{S})}
$$

(because the second parenthesis is 0 by Eq. (25)). Moreover by the definitions of $\widetilde{S}$ and $S^{*}$, the difference $p_{S^{\prime} \cup S}^{*}-\omega_{f\left(S^{\prime} \cup S\right)}$ is not larger for any $S^{\prime} \subseteq \widetilde{S}, S \subseteq M_{+}(p)$, and is strictly smaller if $S$ is not contained in $S^{*}$. This proves the claim. Moreover $M_{-}(p)$ is still a solution of $\arg \max _{S \subseteq M}\left\{p_{S}^{*}-\omega_{f(S)}\right\}$, therefore it is the smallest. So $i \in M_{0}\left(p^{*}\right)$, and $\mathcal{E}_{i}\left(p^{*}\right)=p_{i}^{*}$.

For every $p_{i}^{\prime} \in\left[p_{i}, p_{i}^{*}\left[\right.\right.$, we have $x_{i} \leqslant \mathcal{E}_{i}\left(p^{\prime}\right)$ by Step 1 , hence $x_{i} \leqslant \mathcal{E}_{i}\left(p^{*}\right)$ by continuity of $p_{i}^{\prime} \rightarrow x_{i}$ (explained just before the definition of Strategyproofness). We have shown

$$
p_{i}^{*} \geqslant x_{i} \geqslant p_{i}
$$

Step 3. The proof of Monotonicity for a shift $p_{i} \rightarrow p_{i}^{\prime}$ such that $i \in M_{-}(p)=M_{-}\left(p^{\prime}\right)$ parallels that of Step 1. Similarly if $i \in M_{-}(p)$ there is a critical peak $p_{i}^{*}, p_{i}^{*}<p_{i}$, at which the decomposition changes for the first time. The details of the decomposition at $p^{*}$ are similar and they only matter to prove $i \in M_{0}\left(p^{*}\right)$ and

$$
p_{i}^{*} \leqslant x_{i} \leqslant p_{i}
$$

Step 4. End of proof. We consider now a move from $p_{i}$ to $p_{i}^{\prime}$ when $i \in M_{0}(p)$. If $p_{i}^{\prime}>p_{i}$, we have $i \in M_{-}\left(p^{\prime}\right)$ by Lemma $2\left(M_{+}\right.$is unchanged, and $M_{0}$ shrinks, and may disappear). Then in the downward shift starting at $p_{i}^{\prime}$, the critical value at which the status of $i$ changes is precisely $p_{i}$, so by (27) $x_{i}^{\prime} \geqslant p_{i}=x_{i}$ as desired. Symmetrically $p_{i}^{\prime}<p_{i}$ gives $i \in M_{+}\left(p^{\prime}\right)$ and $p_{i}$ is the critical value in the upward shift starting at $p_{i}^{\prime}$, so (26) gives $x_{i}^{\prime} \leqslant p_{i}=x_{i}$.

To conclude the proof of monotonicity, it remains to look at a shift from $p_{i}$ to $p_{i}^{\prime}$ such that $i \in M_{+}(p)$ and $i \in M_{-}\left(p^{\prime}\right)$. This requires $p_{i}^{\prime}>p_{i}$; clearly the critical value $p_{i}^{*}$ for $p_{i}$ in Step 2 is the same as the critical value for $p_{i}^{\prime}$ in Step 3. Therefore (26) and (27) imply

$$
p_{i} \leqslant x_{i} \leqslant p_{i}^{*} \leqslant x_{i}^{\prime} \leqslant p_{i}^{\prime}
$$

The invariance property is now clear from (26) and (27) and the arguments of Steps 1 and 3.

\section{Characterization result}

Our characterization of the Egalitarian rule generalizes Ching's characterization [7] of the Uniform rule in the standard model.

Theorem 1. The Egalitarian rule is characterized by Pareto Optimality, Strategyproofness and Equal Treatment of Equal Demands.

Proof. We fix $G, \omega$ and a rule $\psi$ meeting the three properties. We show in Step 1 that $\psi=\mathcal{E}$ for "extremists" profiles of demands where agents in $M_{+}$want as little commodity as possible, while those in $M_{-}$want as much as possible. Then in Step 2 we borrow an argument from [7] to extend this equality to all profiles. 
Step 1. We fix in this step two partitions $M_{+,-, 0}$ of $M$ and $Q_{+,-, 0}$ of $Q$, that coincide with the GE decomposition for some profile of peaks $p \cdot{ }^{10}$ Then we choose a profile of preferences $\widetilde{R}$ with peaks $\widetilde{p}$ such that

$$
\begin{aligned}
& \widetilde{R}_{i}=\widetilde{R}_{j} \quad \text { if } i, j \in M_{+} \text {or if } i, j \in M_{-} \\
& \widetilde{p}_{i}=0 \quad \text { if } i \in M_{+} ; \quad \widetilde{p}_{j}>\omega_{Q} \quad \text { if } j \in M_{-} ; \quad\left(G\left(M_{0}, Q_{0}\right), \omega, \widetilde{p}\right) \quad \text { is balanced }
\end{aligned}
$$

We show that $\psi(\widetilde{R})=\mathcal{E}(\widetilde{R})$. Setting $y=\psi(\widetilde{R}), x=\mathcal{E}(\widetilde{R})$, by Proposition 2 it is enough to check that $y^{M_{+}}$(resp. $y^{M_{-}}$) is Lorenz dominant in the corresponding component of $\mathcal{P O}(G, \omega, \widetilde{R})$.

As explained immediately after the statement of Proposition 1 , the $M_{+}$-component of $\mathcal{P O}(G, \omega, \widetilde{R})$ contains $z \geqslant 0$ iff $z$ is in the upper core of the submodular game $\left(M_{+}, v^{+}\right)$, where

$$
v^{+}(S)=\omega_{f(S) \cap Q_{-}} \quad \text { for all } S \subseteq M_{+} ; \quad v^{+}\left(M_{+}\right)=\omega_{Q_{-}}
$$

Similarly the $M_{-}$-component of $\mathcal{P O}(G, \omega, \widetilde{R})$ contains $z$ iff $z$ is in the lower core of the supermodular game $\left(M_{-}, w^{-}\right)$where

$$
w^{-}(S)=\max \left\{\omega_{T} \mid T \subseteq Q_{+}, g(T) \cap M_{-} \subseteq S\right\} \quad \text { for all } S \subseteq M_{-} ; \quad w^{-}\left(M_{-}\right)=\omega_{Q_{+}}
$$

(note that, by our choice of $\widetilde{p}$, the constraints $z \leqslant \widetilde{p}$ are not binding).

We use only Equal Treatment of Equal Demands and Pareto Optimality to show $y^{M_{+}}=x^{M_{+}}$. We omit for brevity the similar argument establishing this equality on $M_{-}$. Set $m_{+}=\left|M_{+}\right|$and recall that $y^{* m_{+}} \geqslant y^{*\left(m_{+}-1\right)} \geqslant \cdots \geqslant y^{* 1}$ is the order statistics of $y$.

Claim 1. Fix an agent $i_{1} \in M_{+}$, such that $y_{i_{1}}=y^{* m_{+}}$; then

$$
y_{i_{1}}=x_{i_{1}}=y^{* m_{+}}=x^{* m_{+}}
$$

Because $x$ Lorenz dominates $y$, we have $y^{* m_{+}} \geqslant x^{* m_{+}}$. If $y_{i}=y^{* m_{+}}$for all $i \in M_{+}$then $y^{M_{+}}=$ $x^{M_{+}}$at once and we are done. If $y_{i_{1}}=0$ (recall in $M_{+}$all peaks are 0 ) then $x_{i_{1}} \geqslant 0$ implies $x_{i_{1}}=y_{i_{1}}$ and (28) is again proven. From now on we assume $y_{i_{1}}>0$, and that there is at least one agent $i$ such that $y_{i}<y^{* m_{+}}$. We show there exists a subset $S(i) \subset M_{+}$such that

$$
i_{1} \notin S(i), \quad i \in S(i), \quad \text { and } \quad y_{S(i)}=v^{+}(S(i))
$$

Otherwise $y_{S}<v^{+}(S)$ for all $S$ in $M_{+}$containing $i$ but not $i_{1}$. Choosing $\varepsilon>0$ smaller than the smallest such difference $v^{+}(S)-y_{S}$, we see that an $\varepsilon$-transfer from agent $i_{1}$ to agent $i$ (a PigouDalton transfer) preserves the core property (inequalities $y_{S} \leqslant v^{+}(S)$ for $S$ containing $i$ are automatically satisfied), and $y_{i_{1}}>0$ ensures the new allocation is nonnegative. This contradiction of (20) proves (29).

Set $S^{*}=\bigcup_{i: y_{i}<y^{* m_{+}}} S(i)$. Submodularity of $v^{+}$implies $y_{S^{*}}=v^{+}\left(S^{*}\right)$, so

$$
x_{S^{*}} \leqslant v^{+}\left(S^{*}\right)=y_{S^{*}} \Rightarrow x_{M_{+} \backslash S^{*}} \geqslant y_{M_{+} \backslash S^{*}}
$$

But by construction $y_{j}=y^{* m_{+}} \geqslant x_{j}$ for all $j \in M_{+} \backslash S^{*}$, therefore $x_{j}=y^{* m_{+}}$for all $j \in$ $M_{+} \backslash S^{*}$. Combining this with $y^{* m_{+}} \geqslant x^{* m_{+}}$proves (28).

Claim 2. Fix an agent $i_{2} \in M_{+}$, such that $i_{2} \neq i_{1}$ and $y_{i_{2}}=y^{*\left(m_{+}-1\right)}$, then

$$
y_{i_{2}}=x_{i_{2}}=y^{*\left(m_{+}-1\right)}=x^{*\left(m_{+}-1\right)}
$$

\footnotetext{
$\overline{10}$ It is easy to check from Lemma 2 that this is possible iff $f\left(M_{-}\right)=Q_{+}, g\left(Q_{-}\right)=M_{+}$, and $f^{-1}\left(Q_{0}\right) \supseteq M_{0}$, $g^{-1}\left(M_{0}\right) \supseteq Q_{0}$.
} 
As $x$ Lorenz dominates $y$, we have $y^{* m_{+}}+y^{*\left(m_{+}-1\right)} \geqslant x^{* m_{+}}+x^{*\left(m_{+}-1\right)} \Rightarrow y^{*\left(m_{+}-1\right)} \geqslant$ $x^{*\left(m_{+}-1\right)}$ (by Claim 1). If $y_{i}=y^{*\left(m_{+}-1\right)}$ for all $i \in M_{+} \backslash i_{1}$ then $y \geqslant x$ so $y=x$ by $y_{M_{+}}=x_{M_{+}}$, and we are done. If $y_{i_{2}}=0$ then $x_{i_{2}} \geqslant 0$ implies $x_{i_{2}}=y_{i_{2}}$ and (30) is again proven. From now on we assume $y_{i_{2}}>0$, and that there is at least one agent $i \in M_{+}$such that $y_{i}<y^{*\left(m_{+}-1\right)}$. For any such agent, we claim there is a subset $S(i) \subset M_{+}$such that

$$
i_{2} \notin S(i), \quad i \in S(i), \quad \text { and } \quad y_{S(i)}=v^{+}(S(i))
$$

Otherwise, we can construct as above a Pigou-Dalton transfer from agent $i_{2}$ to agent $i$, in contradiction of (20). Set $S^{*}=\bigcup_{i: y_{i}<y^{* m_{+}-1}} S(i)$, then submodularity of $v^{+}$gives $y_{S^{*}}=v^{+}\left(S^{*}\right)$, hence

$$
x_{S^{*}} \leqslant v^{+}\left(S^{*}\right)=y_{S^{*}} \Rightarrow x_{M_{+} \backslash S^{*}} \geqslant y_{M_{+} \backslash S^{*}} \Rightarrow x_{M_{+} \backslash\left(S^{*} \cup\left\{i_{1}\right\}\right)} \geqslant y_{M_{+} \backslash\left(S^{*} \cup\left\{i_{1}\right\}\right)}
$$

But by construction $y_{j} \geqslant x_{j}$ for all $j \in M_{+} \backslash\left(S^{*} \cup\left\{i_{1}\right\}\right)$ (as $\left.y_{j} \geqslant y^{* 2}\right)$, and $M_{+} \backslash\left(S^{*} \cup\left\{i_{1}\right\}\right)$ contains $i_{2}$. Combining this with $y^{*\left(m_{+}-1\right)} \geqslant x^{*\left(m_{+}-1\right)}$ proves (28).

The inductive argument establishing $y=x$ is now clear.

Step 2. Extending $\psi=\mathcal{E}$ to all profiles. We fix an arbitrary profile $R^{*} \in \mathcal{R}^{M}$ with peaks $p^{*}$, and associated GE decomposition $M_{+,-, 0}, Q_{+,-, 0}$ of $\left(G, \omega, p^{*}\right)$. We choose $\widetilde{R}$ with peaks $\tilde{p}$ as in Step 1, and the additional requirements $p^{* M_{-}} \leqslant \widetilde{p}^{M_{-}}$and $p^{* M_{0}}=\widetilde{p}^{M_{0}}$; we also have $\widetilde{p}^{M_{+}}=0 \leqslant p^{* M_{+}}$.

Given $S \subset M$, we write $\left(R^{* S}, \widetilde{R}^{(M \backslash S)}\right)$ for the profile equal to $R^{*}$ for agents in $S$ and to $\widetilde{R}$ for agents in $M \backslash S$. For any integer $n, 0 \leqslant n \leqslant m$, consider the following subset of preference profiles

$$
\mathcal{D}_{n}=\left\{\left(R^{* S}, \widetilde{R}^{(M \backslash S)}\right) \mid \text { for some } S \subset M:|S| \leqslant n\right\}
$$

We prove by induction on $n$ the property $\mathcal{H}^{+}(n): \psi=\mathcal{E}$ on $\mathcal{D}_{n}$. This is enough because Step 1 establishes $\mathcal{H}^{+}(0)$, and $\mathcal{H}^{+}(m)$ means $\psi\left(R^{*}\right)=\mathcal{E}\left(R^{*}\right)$ for an arbitrary $R^{*}$.

Assume $\mathcal{H}^{+}(n-1)$ is true, and fix $R=\left(R^{* S}, \widetilde{R}^{(M \backslash S)}\right)$ with $|S|=n$. We claim $\psi(R)^{S \cap M_{+}}=$ $\mathcal{E}(R)^{S \cap M_{+}}$. Pick an arbitrary agent $i \in S \cap M_{+}$. By Pareto Optimality $p_{i}^{*} \leqslant \psi_{i}(R), \mathcal{E}_{i}(R)$. To prove $\psi_{i}(R)=\mathcal{E}_{i}(R)$ we consider the profile $R^{\prime}=\left(R^{*(S \backslash i)}, \widetilde{R}^{(M \backslash S) \cup\{i\}}\right) \in \mathcal{D}_{n-1}$ where the inductive assumption gives $\psi_{i}\left(R^{\prime}\right)=\mathcal{E}_{i}\left(R^{\prime}\right)=z_{i}$. We compare $\psi_{i}(R), \mathcal{E}_{i}(R)$ and $z_{i}$ by distinguishing two cases.

If $p_{i}^{*} \leqslant \psi_{i}(R)<\mathcal{E}_{i}(R)$ then $z_{i} \leqslant \psi_{i}(R)$ by Monotonicity of $\psi$ (Lemma 3 ) and $\widetilde{p}^{M_{+}} \leqslant p^{* M_{+}}$. As $\mathcal{E}$ is Invariant (Proposition 4$)$ and $\widetilde{p}_{i}, p_{i}^{*}<\mathcal{E}_{i}\left(R_{i}^{*}, R_{-i}\right)$, we have $\mathcal{E}_{i}\left(\widetilde{R}_{i}, R_{-i}\right)=\mathcal{E}_{i}\left(R_{i}^{*}, R_{-i}\right)$, i.e., $z_{i}=\mathcal{E}_{i}(R)$. This is a contradiction. If $p_{i}^{*} \leqslant \mathcal{E}_{i}(R)<\psi_{i}(R)$ the same contradiction obtains by exchanging the role of $\psi$ and $\mathcal{E}$. The claim is proven.

We check next $\psi(R)^{M_{+} \backslash S}=\mathcal{E}(R)^{M_{+} \backslash S}$. Write $\psi(R)^{M_{+}}=y, \mathcal{E}(R)^{M_{+}}=x$, and consider the set

$$
\mathcal{C}(R)=\left\{z \in \mathbb{R}_{+}^{M_{+} \backslash S} \mid\left(z, x^{S \cap M_{+}}\right) \text {is in the upper core of }\left(M_{+}, v^{+}\right)\right\}
$$

It contains $y^{M_{+} \backslash S}$ because $y^{S \cap M_{+}}=x^{S \cap M_{+}}$. Clearly $x^{M_{+} \backslash S}$ is still Lorenz dominant in $\mathcal{C}(R)$, hence we can mimic the proof of Step 1 to show that Equal Treatment of Equal Demands and Pareto Optimality imply the desired equality of $x^{M_{+} \backslash S}$ and $y^{M_{+} \backslash S}$. The key is that the restriction of the profile $\widetilde{R}$ to $M_{+} \backslash S$ consists of pairwise identical preferences, therefore we can apply Equal Treatment of Equal Demands to any pair of agents in $M_{+} \backslash S$. To copy the proof of Step 1, observe that $\mathcal{C}(R)$ is defined, besides the constraints $z \geqslant 0$, by the inequalities 


$$
z_{A} \leqslant \widehat{v}^{+}(A)=v^{+}\left(A \cup\left(S \cap M_{+}\right)\right)-x_{S \cap M_{+}} \text {for all } A \subset M_{+} \backslash S
$$

and the equality $z_{M_{+} \backslash S}=x_{M_{+} \backslash S}$. Thus $\mathcal{C}(R)$ is the upper core of the submodular game $\left(M_{+} \backslash S, \widehat{v}^{+}\right)$and the proof proceeds exactly as in Step 1 . We omit the details.

We also omit the entirely similar proof that $\psi_{i}(R)=\mathcal{E}_{i}(R)$ on $M_{-}$.

\section{Concluding comments}

We first list four more normative requirements for our model, three of which are satisfied by the Egalitarian rule.

1) Group-Strategyproofness strengthens Strategyproofness by ruling out profitable joint misreports by arbitrary subsets of agents. In the standard model it is well known that the Uniform rule is group-strategyproof and Barbera et al. [2] offer a group-strategyproof allocation rule which may treat agents unequally. Chandramouli and Sethuraman [6] recently established that the present Egalitarian rule is group-strategyproof as well.

2) Resource Monotonicity requires that the share of every agent increases weakly when the amount of one of the resources (one of the numbers $\omega_{r}$ ) increases [27]. For instance we go from Fig. 1 (Section 2) to Fig. 2 (Section 4) by adding 2 units to resource $s$, and the Egalitarian solution goes from $(11,15,9,5)$ in Fig. 1 to $(13,15,9,5)$ in Fig. 2. Our Egalitarian rule is Resource Monotonic. The proof mimics that of the analog "Peak Monotonicity" property in the companion paper [4]; it is omitted for brevity.

3) Consistency plays a central role in characterizing the parametric rules of the standard model (these include the Uniform rule and many more): see [30] and [29]. It can be adapted to our model in two ways, by dropping an agent or dropping a resource (see [18] for a formal definition). Consider a rule $\psi$, a problem $(M, G, \omega, R)$, and a $G$-flow $\varphi$ implementing $\psi(M, G, \omega, R)$. If agent $i$ leaves with her share of $\varphi$, we delete from $G$ all edges between $i$ and $Q$, and reduce the endowment of resource $r$ to $\omega_{r}(-i)=\omega_{r}-\varphi_{i r}$. Agent-Consistency of $\psi$ requires that $\varphi(-i)$ implements the allocation $\psi\left(M \backslash i, G(-i), \omega(-i), R_{-i}\right)$. From its Lorenz dominance property, it is clear that the Egalitarian solution is agent-consistent.

Symmetrically, Resource-Consistency considers dropping a resource $r$, deleting from $G$ all edges between $M$ and $r$, and shifting the preferences of agent $i$ by $\varphi_{i r}$, i.e., the flow that agent $i$ gets from resource $r$ at $\psi(M, G, \omega, R)$. Her peak then becomes $\left(p_{i}-\varphi_{i r}\right)$ if $p_{i}-$ $\varphi_{i r}>0$, and 0 otherwise. The preference relation $R_{i}\left(-\varphi_{i r}\right)$ resulting from this operation is the obvious translation of $R_{i}$ around the new peak $\left(p_{i}-\varphi_{i r}\right)$ if $p_{i}-\varphi_{i r}>0$, or the always decreasing preference if $p_{i}-\varphi_{i r} \leqslant 0$. Resource-Consistency requires that $\varphi(-r)$ implements the solution $\psi(M, G(-r), \omega(-r), R(-r))$. The Egalitarian rule fails this property, as one sees in the examples of Fig. 1 and Fig. 2 by dropping resources $r, s, t$. The reduced oneresource problem has $C$ with peak 2 , and $D$ with peak 5, sharing 6 units: the Uniform solution is $\tilde{x}_{C}=2, \widetilde{x}_{D}=4$, whereas the Egalitarian solution of the initial problem gives only one unit of resource $u$ to $C$, and $x_{D}=5$.

As mentioned in the introduction, the follow-up paper [18] focuses on rules meeting both versions of Consistency. In particular the standard Uniform rule admits infinitely many consistent extensions to the bipartite model.

4) Edge Monotonicity: what happens when we drop or add an edge? Suppose agent $i$ is rationed ( $i \in M_{-}$), and we are adding an edge to an overdemanded resource $r$ in $Q_{+}$: we expect $i$ to benefit (weakly) while other rationed agents are weakly hurt. This is indeed the case, 
and follows from Proposition 4 in [4]. However if the new edge connects $i$ to an underdemanded resource $r^{\prime}$ in $Q_{-}$, the impact on $i$ can be good or bad: he is now able to relieve the overloaded agents in $M_{+}$of some of their burden, so he may end up overloaded himself. ${ }^{11}$

We discuss finally three extensions of our model.

5) We can add capacity constraints to the total allocation of each agent. Fix $c^{-}, c^{+} \in \mathbb{R}_{+}^{M}$ such that $c^{-} \leqslant c^{+}$, and insist that an allocation $x \in \mathbb{R}_{+}^{M}$ is feasible only if $c_{i}^{-} \leqslant x_{i} \leqslant c_{i}^{+}$. Combining this with the bipartite constraints, the set of feasible allocations becomes $\mathcal{A}(G, \omega) \cap\left[c^{-}, c^{+}\right]$. This set is nonempty if and only if

$$
\text { for all } S \subseteq M, \quad c_{S}^{-} \leqslant \omega_{f(S)} ; \quad \text { and } \quad \text { for all } T \subseteq Q, \quad \omega_{T} \leqslant c_{g(T)}^{+}
$$

an assumption we must maintain. Then the "core" representations of feasible allocations, statements iv) and v) in Lemma 1 , are easily adapted. ${ }^{12}$ Preferences of agent $i$ bear only on $\left[c_{i}^{-}, c_{i}^{+}\right]$, so the profile of peaks $p$ is in $\left[c^{-}, c^{+}\right]$. The GE decomposition (Lemma 2) is unchanged, and so is the description of Pareto optimal allocations (Proposition 1), except for the addition of the capacity constraints. For instance in $M_{+}$the constraints become $x^{M_{+}} \in$ $\mathcal{A}\left(G\left(M_{+}, Q_{-}\right), \omega\right)$, and $p^{M_{+}} \leqslant x^{M_{+}} \leqslant c^{+}$: they are compatible because the system (31) is true when $p$ replaces $c^{-}$.

To define the Egalitarian solution in $M_{+}$, we use the same system $\Theta(\lambda)$ but with the median function $\gamma_{i}(\lambda)=\operatorname{med}\left\{\lambda, p_{i}, c_{i}^{+}\right\}$, guaranteeing that $\gamma_{i}(\lambda)$ remains below $c_{i}^{+}$. Similarly in system $\Xi(\mu)$ we set $\delta_{i}(\mu)=\operatorname{med}\left\{\mu, c_{i}^{-}, p_{i}\right\}$. The Lorenz dominant position of $\mathcal{E}(G, \omega, p, c)$ in the Pareto set (Proposition 2) follows from the same argument, and so does the proof that the Egalitarian rule is Strategyproof (Proposition 4).

On the other hand, the properties of Equal Treatment of Equals, and No Envy have much less bite in the presence of arbitrary capacity constraints. Two agents with disjoint capacity ranges $\left(\left[c_{i}^{-}, c_{i}^{+}\right] \cap\left[c_{i}^{-}, c_{i}^{+}\right]=\emptyset\right)$ cannot envy one another, nor can we talk of their preferences being equal. So our Egalitarian rule passes the version of these two axioms applying only to overlapping (or even equal) capacity ranges, but this is not enough to extend the characterization result.

6) There is a "discrete" variant of the standard model where indivisible units have to be distributed. Two papers [22,12] characterize in this case the randomized Uniform rule for the standard model. It is possible that their result could be adapted to include bilateral constraints.

7) We have considered only symmetric rules. In the standard model, the rich family of allotment rules [2] preserves the incentive properties of the Uniform rule while allowing a very different treatment of the agents. Similarly the family of fixed paths rules [17] is characterized by the combination of Pareto Optimality, Strategyproofness, Resource Monotonicity and Consistency. Both families can be extended to our model, though the corresponding characterization results, if any, would require further research.

\footnotetext{
11 In the example of Fig. 2 the rule $\mathcal{E}$ selects $\left(x_{A}, x_{B}, x_{C}, x_{D}\right)=(13,15,9,5)$. Adding an edge from agent $C$ to resource $r$ yields a new problem where all resources are underdemanded $\left(M=M_{+}, Q=Q_{-}\right)$, and the new Egalitarian allocation is $(10.5,15,10.5,6)$. Agent $A$ strictly benefits in the change, while $D$ is hurt. If $9 P_{C} 10.5$, agent $C$ is hurt as well.

$12 x \in \mathcal{A}(G, \omega) \cap\left[c^{-}, c^{+}\right] \Leftrightarrow\left\{x \leqslant c^{+}\right.$and $x$ is in the lower core of the supermodular game $(M, w)$, where $w(S)=$ $\left.\max _{T: g(T) \subseteq S}\left\{\omega_{T}+c_{S \backslash g(T)}^{-}\right\}\right\} \Leftrightarrow\left\{x \geqslant c^{-}\right.$and $x$ is in the upper core of the submodular game $(M, v)$, where $v(S)=$ $\left.\min _{S^{\prime} \subseteq S}\left\{\omega_{f\left(S^{\prime}\right)}+c_{S \backslash S^{\prime}}^{+}\right\}\right\}$.
} 


\section{References}

[1] R.K. Ahuja, T.L. Magnati, J.B. Orlin, Network Flows: Theory, Algorithms and Applications, Prentice Hall, 1993.

[2] S. Barbera, M.O. Jackson, A. Neme, Strategy-proof allotment rules, Games Econ. Behav. 18 (1997) 1-21.

[3] J.-P. Benassy, The Economics of Market Disequilibrium, Academic Press, New York, 1982.

[4] O. Bochet, R. İlkılıç, H. Moulin, J. Sethuraman, Clearing supply and demand under bilateral constraints, Theoretical Econ. 7 (2012) 395-424.

[5] A. Bogomolnaia, H. Moulin, Random matching under dichotomous preferences, Econometrica 72 (2004) 257-279.

[6] S. Chandramouli, J. Sethuraman, Groupstrategyproofness of the egalitarian mechanism for constrained rationing problems, mimeo, Columbia University, 2011.

[7] S. Ching, An alternative characterization of the uniform rule, Soc. Choice Welfare 40 (1994) 57-60.

[8] T. Cormen, C. Leiserson, R. Rivest, C. Stein, Introduction to Algorithms, MIT Press, 2001.

[9] M. Fleurbaey, Fairness, Responsibility, and Welfare, Oxford University Press, 2008.

[10] A. De Frutos, J. Masso, More on the uniform allocation rule: equality and consistency, WP 288.95 UAB, 1995.

[11] B. Dutta, D. Ray, A concept of egalitarianism under participation constraints, Econometrica 57 (1989) $615-635$.

[12] L. Ehlers, B. Klaus, Probabilistic assignments of identical indivisible objects and uniform probabilistic rules, Rev. Econ. Design 8 (2003) 249-268.

[13] J. Foster, A. Sen, On Economic Inequality, Clarendon Press, Oxford, 1973.

[14] P. Hall, On representatives of subsets, J. London Math. Soc. 10 (1935) 26-30.

[15] A. Kar, O. Kıbrıs, Allocating multiple estates among agents with single-peaked preferences, Soc. Choice Welfare 31 (2008) 641-666.

[16] H. Moulin, Axioms of Cooperative Decision Making, Econom. Soc. Monogr., Cambridge University Press, Cambridge, 1988.

[17] H. Moulin, Rationing a commodity along fixed paths, J. Econ. Theory 84 (1999) 41-72.

[18] H. Moulin, J. Sethuraman, The bipartite rationing problem, mimeo, Columbia University, Rice University, 2011.

[19] O. Ore, Theory of Graphs, Amer. Math. Soc. Colloq. Publ., vol. XXXVIII, American Mathematical Society, Providence, RI, 1962.

[20] J. Roemer, Theories of Distributive Justice, Harvard University Press, 1996.

[21] R. Roth, T. Sönmez, U. Ünver, Pairwise kidney exchange, J. Econ. Theory 125 (2005) 151-188.

[22] H. Sasaki, Randomized uniform allocation mechanism and single-peaked preferences of indivisible good, mimeo, Waseda University, 1997.

[23] J. Schummer, W. Thomson, Two derivations of the uniform rule and an application to bankruptcy, Econ. Letters 55 (1997) 333-337.

[24] A. Sen, Collective Choice and Social Welfare, Holden-Day, San Francisco, 1970.

[25] Y. Sprumont, The division problem with single-peaked preferences: a characterization of the uniform allocation rule, Econometrica 59 (1991) 509-519.

[26] K. Szwagrzak, The replacement principle in networked economies with single-peaked preferences, mimeo, University of Rochester, 2011.

[27] W. Thomson, Resource monotonic solutions to the problem of fair division when preferences are single-peaked, Soc. Choice Welfare 63 (1994) 205-224.

[28] W. Thomson, The replacement principle in economies with single-peaked preferences, J. Econ. Theory 76 (1997) $145-168$.

[29] W. Thomson, Consistent solutions to the problem of fair division when preferences are single-peaked, J. Econ. Theory 63 (1994) 219-245.

[30] H.P. Young, On dividing an amount according to individual claims or liabilities, Math. Oper. Res. 12 (1987) 397414. 